\title{
Network-Coded Cooperative Systems With Generalized User-Relay Selection
}

\author{
Ali Reza Heidarpour, Student Member, IEEE, Masoud Ardakani, Senior Member, IEEE, Chintha \\ Tellambura, Fellow, IEEE, Marco Di Renzo, Fellow, IEEE, and Murat Uysal, Fellow, IEEE
}

\begin{abstract}
We consider a network-coded cooperative (NCC) system that consists of $N \geq 2$ sources, $M \geq 1$ decode-andforward (DF) relays, and a single destination. The relays perform network coding (NC) on the received sources' symbols using maximum distance separable (MDS) codes. For this system, we propose the most generalized user-relay selection (GURS) scheme in the literature that selects any arbitrary subsets of $K$ users and any arbitrary subsets of $L$ relays subject to practical constraints such as load balancing conditions and scheduling policy. Our analytical results and design guidelines generalize and subsume all existing results as special cases. To this end, we derive a new closed-form outage probability (OP) expression, assuming non-identically and independently distributed (n.i.i.d.) Rayleigh fading channels. The asymptotic outage expression at high signalto-noise ratio (SNR) regime is further derived, based on which, the achievable diversity order and coding gain are quantified. The theoretical derivations are also validated through Monte-Carlo simulation.
\end{abstract}

Keywords-Generalized user-relay selection (GURS), networkcoded cooperative, outage analysis, diversity order.

\section{INTRODUCTION}

$\mathbf{T}$ HE next generation of wireless communication systems are expected to offer high data rate, ultra-reliable low latency, and improved energy efficiency. Fifth generation (5G) wireless technologies are under development. 5G can handle billions of heterogeneous devices while providing very high data rates at very low latency. International telecommunication union (ITU) has specified a roadmap for $5 \mathrm{G}$ in term of 'IMT-2020'. IMT-2020 vision aims to deliver up to $10-\mathrm{Gbps}$ peak data rates, $1 \mathrm{~ms}$ over-the-air latency, and $100 \times$ energy efficiency improvement [1].

Cooperative communication, also called cooperative diversity (CD), is a promising candidate to fulfill the requirements for wireless technology 2020 and beyond. CD exploits spatial diversity by forming a virtual antenna array through the spatially distributed relay nodes [2]. Cooperative relay networks have been thus adopted by several wireless system standards

This work was supported by the Telus Communications Inc. and Alberta Innovates Graduate Student Scholarship.

A. R. Heidarpour, M. Ardakani, and C. Tellambura are with the Department of Electrical and Computer Engineering, University of Alberta, Edmonton, AB, T6G 1H9, Canada (e-mail: \{alirezaheidarpour, ardakani, ct4\}@ualberta.ca).

M. Di Renzo is with Université Paris-Saclay, CNRS and CentraleSupélec, Laboratoire des Signaux et Systèmes, Gif-sur-Yvette, France. (email: marco.direnzo@centralesupelec.fr).

Murat Uysal is with the Department of Electrical and Electronics Engineering, Ozyegin University, Istanbul 34794, Turkey (e-mail: murat.uysal@ozyegin.edu.tr).

This paper was presented in part at the IEEE International Conference on Communications (ICC), Shanghai, China, May 2019.
[3] and are also considered as a key enabler technology for future wireless communications [4].

The traditional repetition-based CD protocols, however, entail the loss of system throughput since each relay transmits sources' packets to the destination over orthogonal channels. This spectral inefficiency is prohibitive, especially in cooperative networks with a large number of sources.

Network-coded cooperation (NCC), a new class of CD protocols, is a solution to this inefficiency in CD systems [5]. Thus, NCC avoids separately re-transmitting each source packet; instead, each relay employs network coding (NC) [6] to combine packets received from multiple sources; generates a new network-coded packet; and then transmits it to the destination. This process decreases the number of channel uses required by the relays and thus increases the system throughput.

\section{A. Related Literature}

The design and analysis of NCC, in general, build upon two error propagation models: i) the erasure channel model; and ii) error channel model. In the former, the erroneous sources' packets are discarded at the relays and thus no error propagation occurs ${ }^{1}$. In the latter, however, the erroneous packets are allowed to propagate through the network, but error propagation is counteracted at the destination with the aid of appropriate "error-aware" demodulators. In what follows, we will provide a summary of research works under these two channel models.

The performance analysis of NCC under the erasure channel model has been studied in [7]-[11]. In particular, the network code design for generic multisource multirelay networks with a single destination was studied in [7]. It is shown that binary $\mathrm{NC}$ is not diversity optimal, while a non-binary $q$ ary Galois field $\mathrm{NC}$ based on maximum distance separable (MDS) codes provides the full diversity order irrespective of the number of sources and relays. The outage probability (OP) and diversity-multiplexing tradeoff (DMT) of NCC have been also studied in [8]-[10], demonstrating that NCC provides a better DMT compared to the traditional CD protocols. The effect of outdated channel state information (CSI) on the performance of relay selection (RS) based NCC has been further investigated in [11].

Several other seminal works have also studied the performance of NCC under error channel model. For instance, [12], [13] investigated the performance of NCC with binary modulation and binary NC. In particular, the average bit

\footnotetext{
${ }^{1}$ This can be implemented in practice using a cyclic redundancy check (CRC).
} 
error probability (ABEP) was computed for two types of demodulaters; a hard decision based demodulator [12] and a NCC maximal ratio combining (MRC) demodulator [13]. Furthermore, guidelines for network code design were developed and the impact of error propagation on the diversity order and coding gain was quantified. Later, the performance of repetition-based and RS-based NCC protocols were investigated in [14], assuming NCC-MRC demodulator and arbitrary modulation order and arbitrary Galois field size. Following [14], the exact and asymptotic expressions of the OP for single RS and multiple RS protocols were further derived in [15]. The diversity analysis in [14], [15] reveals that RS-based NCC achieves full diversity order under a restrictive condition where the number of selected relays must be at least equal to the number of sources. It is further shown that the design of full diversity-achieving network codes is identical to that of linear block codes based on MDS codes [14]. Finally, in [16], the authors extended the results in [14], [15] by studying the performance of RS in multiple-input multiple-output (MIMO) NCC systems.

\section{B. Motivation and Contributions}

Multiuser diversity (MUD) is inherent in a network of spatially separated users and thus provides a form of diversity against fading. The basic premise of MUD is to exploit channel variations among those users by allocating resources to the best users experiencing good channel qualities. The application of MUD to conventional CD has been studied [17]-[19], demonstrating that MUD-based CD with RS exploits both MUD and CD gains and thus offers substantial performance improvement.

The application of MUD to NCC has tremendous potential to further improve the the performance of NCC systems. However, so far the performance analysis of such a system has been only investigated in [20]. In particular, [20] is the first paper that studies the performance of MUD-based NCC under the erasure channel model ${ }^{2}$. One of the main contributions of this work is to add to this literature and study the performance of MUD-based NCC, assuming the error channel model. Therefore, our analytical expressions are new and different from those presented in [20].

Effective resource allocation strategies are key design considerations in $5 \mathrm{G}$ and future cellular networks. From a spectral efficiency perspective, the best solution is to allocate a resource block to the best user experiencing the highest SNR, which maximizes the cell throughput. Scheduling based on maxSNR, however, does not account other important factors such as fairness, cell-edge coverage, and energy efficiency. This necessitates a flexible resource allocation mechanism that provides a good trade-off among different performance objectives (e.g. throughput, delay, or energy). On the other hand, traffic load disparity, inherited from scheduling schemes based on the max-SNR criterion, inevitably leads to suboptimal resource allocations across the network, particularly in Long Term Evolution-Advanced (LTE-Advanced) heterogeneous networks

\footnotetext{
${ }^{2}$ The authors in [20] proposed a user-relay selection that selects the best users and relays to exploit both MUD and CD gains in NCC systems.
}

(HetNets) with diverse quality of service (QoS) requirements. Under these circumstances, user/relay selection may be based on factors other than SNR. Furthermore, there are several practical scenarios that selecting the best-SNR users/relays might be inefficient or even infeasible. For instance, the scheduler may fail to select the best users/relays in the presence of imperfect CSI; the best-SNR users may not have any data packet to transmit; and the best-SNR users/relays might run out of the battery at the time of transmission. These observations suggest that the ability to select an arbitrary set of users and/or relays is beneficial. Thus, the performance of generalized user selection [21]-[23] and generalized RS [24]-[27] have been separately and extensively studied recently. However, the performance analysis of generalized user-relay selection (GURS) has not been investigated in the literature. The objective of this paper is to remedy this gap. We propose the most GURS scheme that selects any arbitrary subsets of users and any arbitrary subsets of relays subject to any practical constraints. Our analysis evaluates the performance loss incurred when suboptimal user-relay selection is performed and thus provides the basis for better scheduling and efficient resource management algorithms in $5 \mathrm{G}$ and future cellular networks.

In particular, we consider a dual-hop multiuser multirelay cooperative network that consists of $N \geq 2$ sources, $M \geq 1$ relays, and a single destination. The relays use decode-andforward (DF) relaying and apply NC on received sources' symbols, that are either correctly or incorrectly demodulated, using the weighting coefficients forming an MDS code. The proposed user-relay selection mechanism selects any arbitrary subsets of $K$ (out of $N$ ) users and any arbitrary subsets of $L$ (out of $M$ ) relays subject to any practical constraints such as load balancing conditions, scheduling policy, and other factors.

The main contributions of the present paper are summarized as follows:

- New closed-form OP expression is derived, assuming non-identically and independently distributed (n.i.i.d.) Rayleigh fading over all the wireless links ${ }^{3}$.

- We derive a concise high signal-to-noise ratio (SNR) OP expression, based on which, the achievable diversity order and coding gain are quantified; two system-design parameters that govern the OP in the high-SNR regime.

- We show that our performance analysis and design guidelines are applicable to a large set of situations and generalize and subsume all existing results in the literature as special cases.

- We further confirm our theoretical findings through extensive Monte-Carlo simulations.

\section{Organization and Notations}

The rest of this paper is organized as follows: Section II describes the system and channel models. Section III presents

\footnotetext{
${ }^{3} 5 \mathrm{G}$ wireless communication systems use two main frequency bands, namely traditional sub- $6 \mathrm{GHz}$ band $(<6 \mathrm{GHz})$ and millimeter-wave $(\mathrm{mm}-$ wave) band (30-300 GHz). In this paper, we consider sub- $6 \mathrm{GHz} 5 \mathrm{G}$ and assume n.i.i.d. Rayleigh fading channels to model multipath fading environments. The Rayleigh fading model for the sub- $6 \mathrm{GHz}$ band, however, cannot be applied to mm-wave band due to poor scattering propagation and significant attenuation of mm-wave signals.
} 


\begin{tabular}{|c|c|c|c|c|c|c|c|c|}
\hline \multicolumn{5}{|c|}{ Broadcasting Phase } & \multicolumn{4}{|c|}{ Relaying Phase } \\
\hline $\begin{array}{c}S_{\left(i_{1}\right)} \\
\text { transmits }\end{array}$ & $\begin{array}{c}S_{\left(i_{2}\right)} \\
\text { transmits }\end{array}$ & $\cdots$ & $\begin{array}{l}1 \\
1 \\
1 \\
1\end{array}$ & $\begin{array}{c}S_{\left(i_{K}\right)} \\
\text { transmits }\end{array}$ & $\begin{array}{c}R_{\left(j_{1}\right)} \\
\text { transmits }\end{array}$ & $\begin{array}{l} \\
\text { I transmits } \\
\end{array}$ & $\cdots$ & $\begin{array}{c}R_{\left(j_{L}\right)} \\
\text { transmits }\end{array}$ \\
\hline
\end{tabular}

$K+L$ time-slots

Fig. 1. Timing diagram for GURS NCC.

the detailed analysis of outage performance of GURS NCC. Asymptotic analysis is provided in Section IV. Numerical results are presented in Section V. Finally, we conclude in Section VI.

Notations: Throughout this paper, the following notations are used: $\operatorname{Pr}\{A\}$ and $\left(\begin{array}{l}n \\ k\end{array}\right)=\frac{n !}{(n-k) ! k !}$ denote the probability of an event $A$ and the binomial coefficient, respectively. $\mathbb{F}_{q}$ denotes Galois field with size $q$. Addition and multiplication in $\mathbb{F}_{q}$ are denoted by $\oplus$ and $\otimes$, respectively.

\section{System MOdEL AND TRANSMISSION SCHEME}

In this section, we first explain the system and channel models and thereafter describe the signal model and transmission protocol in detail.

\section{A. System and Channel Models}

Consider a dual-hop multiuser multirelay network with $N$ sources $\mathcal{S}=\left\{S_{n}\right\}_{n=1}^{N}$, one destination $D$, and $M$ DF relays $\mathcal{R}=\left\{R_{m}\right\}_{m=1}^{M}$. The direct links between sources and the destination are available, and the relays assist the sources to deliver the information packets to the destination. Each node is equipped with a single antenna, transmits with power $\rho$, and operates in the half-duplex fashion. The transmissions occur in different orthogonal time-slots and the cooperation takes place in two phases, namely i) the broadcasting phase; and ii) the relaying phase. Fig. 1 depicts the timing diagram for GURS NCC system. In the broadcasting phase, the $i_{1}^{\text {th }}, i_{2}^{\text {th }}, \ldots, i_{K}^{\text {th }}$ best sources $\left\{S_{\left(i_{k}\right)}\right\}_{k=1}^{K}$ (amongst $N$ sources) are selected to transmit their messages to the destination in a round-robin fashion. The source selection might include a set of $K$ highestSNR sources or any other possible selection. This phase lasts $K$ time-slots. Thanks to the broadcast nature of the wireless medium, the $M$ relays also overhear the transmissions. In the relaying phase, any arbitrary subset of relays of size $L$ (out of $M$ available relays), the $j_{1}^{\text {th }}, j_{2}^{\text {th }}, \ldots, j_{L}^{\text {th }}$ best relays $\left\{R_{\left(j_{l}\right)}\right\}_{l=1}^{L}$, can be selected. More specifically, the selected $L$ relays employ NC to linearly combine $K$ received packets and then are assigned orthogonal channels to sequentially forward the resulting network-coded packets to the destination. This phase thus takes place in $L$ time-slots.

The network subchannels are subjected to independent slow and frequency non-selective Rayleigh fading. We consider independent but non-identically distributed (i.e., asymmetric) Rayleigh fading channels. In particular, the channel coefficient of link $i \rightarrow j$ is denoted by $h_{i j}$ and follows $h_{i j} \sim \mathcal{C N}\left(0, \sigma_{i j}^{2}\right)$; a circularly-symmetric complex Gaussian random variable (RV) whose mean is zero and whose variance is equal to $\sigma_{i j}^{2}$. Furthermore, the additive white Gaussian noise (AWGN) term of link $i \rightarrow j$ is denoted by $w_{i j}$ and has mean zero and unit variance i.e., $w_{i j} \sim \mathcal{C N}(0,1)$. We remark that although the transmit power and the noise variance are set to be symmetric throughout the network, asymmetry cases can be lumped into the fading variances.

In GURS NCC, $\left(\begin{array}{c}N \\ K\end{array}\right)\left(\begin{array}{c}M \\ L\end{array}\right)$ different source-relay selections are possible. It is customary to assume a centralized selection method where the source-relay selection process is performed by a central unit (this could also be the destination). This entity requires instantaneous CSI of the source-to-destination links for source selection, while it requires the CSI of source-torelay and relay-to-destination links for RS process ${ }^{4}$. The CSI of the indirect source-to-relay links are estimated by the relays using pilot sequences sent by selected $K$ sources and then are forwarded to the destination. The selection depends on load balancing conditions, scheduling policy, and other factors.

\section{B. Signal Model and Transmission Scheme}

1) Broadcasting Phase: In this phase, the destination selects sources $\left\{S_{\left(i_{k}\right)}\right\}_{k=1}^{K}$ for data transmission. The source selection criterion is based on the instantaneous SNR of the direct source-to-destination links. We define $\gamma_{(n)}$ as the $n^{\text {th }}$ largest SNR of the source-to-destination SNRs. Specifically, $\gamma_{(n)}$ can be written as

$$
\gamma_{(n)}=n^{\text {th }} \max _{1 \leq n \leq N}\left\{\gamma_{S_{n} D}\right\} .
$$

Let $\left\{\gamma_{\left(i_{k}\right)}\right\}_{k=1}^{K}$ denote the ordered SNRs of any arbitrary subset of $\left\{\gamma_{(n)}\right\}_{n=1}^{N}$ and $\mathcal{I}=\left\{i_{k}\right\}_{k=1}^{K}$ being the set of indexes of the elements in $\left\{\gamma_{\left(i_{k}\right)}\right\}_{k=1}^{K}$ where $i_{1}<i_{2}<\ldots<i_{K}$. For the special case when the source selection includes the $K$ highest-SNR sources, we have $\mathcal{I}=\{1,2, \ldots, K\}$.

Denoting $\epsilon_{S_{(k)}} \in \mathbb{F}_{q}$ as the symbol transmitted by the selected source $S_{(k)}, k \in \mathcal{I}$, the received signal at relay $R_{m}$ $(\forall m)$ and $D$ can be expressed as

$$
\begin{gathered}
y_{S_{(k)} D}=\sqrt{\rho} h_{S_{(k)} D} x_{S_{(k)}}+w_{S_{(k)} D}, \\
y_{S_{(k)} R_{m}}=\sqrt{\rho} h_{S_{(k)} R_{m}} x_{S_{(k)}}+w_{S_{(k)} R_{m}},
\end{gathered}
$$

where $x_{S_{(k)}}$ is the modulated version of $\epsilon_{S_{(k)}}$.

2) Relaying Phase: This phase is based on the RS policy, which minimizes the possible error of network-coded symbols [14]. Under this selection strategy, the equivalent channel for relay $R_{m}$ is determined by the worst channel in the two-hop source-relay-destination links. Let $\mathcal{A}$ denote the set of indexes of the selected sources. The cardinality of $\mathcal{A}$ is $K$ and the

\footnotetext{
${ }^{4}$ In time-varying fading channels, due to a delayed feedback the instantaneous CSI used in user/relay selection may substantially differ from the CSI at the data transmission instant. The outdated CSI may result in wrong selections, and hence impact the system performance. The effect of the outdated CSI on the performance of GURS NCC is an interesting research top and is left as a future work.
} 
number of all possible $\mathcal{A}$ 's is $\left(\begin{array}{l}N \\ K\end{array}\right)$. The "equivalent SNR" of the channels between $K$ selected sources, relay $R_{m}$, and the destination can then be expressed as

$$
\gamma_{m \mid \mathcal{A}}^{\min }=\min \left\{\gamma_{S_{\left(i_{1}\right)} R_{m}}, \gamma_{S_{\left(i_{2}\right)} R_{m}}, \ldots, \gamma_{S_{\left(i_{K}\right)} R_{m}}, \gamma_{R_{m} D}\right\} .
$$

Define $g_{(m)}$ as the $m^{\text {th }}$ largest equivalent SNRs of relays. Mathematically, this can be expressed as

$$
g_{(m)}=m^{\text {th }} \max _{1 \leq m \leq M}\left\{\gamma_{m \mid \mathcal{A}}^{\min }\right\} .
$$

In the relaying phase, relays $\left\{R_{\left(j_{l}\right)}\right\}_{l=1}^{L}$ take part in cooperation. Let $\left\{g_{\left(j_{l}\right)}\right\}_{l=1}^{L}$ denote the ordered SNRs of any arbitrary subset of $\left\{g_{(m)}\right\}_{m=1}^{M}$, where $j_{1}<j_{2}<\ldots<j_{L}$. As an example, assume that the number of relays $M=10$ and $\mathcal{J}=\left\{j_{l}\right\}_{l=1}^{L}=\{1,3,7,9\}$. This implies that four relays out of ten relays are selected whose SNRs are the first, third, seventh, and ninth largest SNRs in $\left\{g_{(m)}\right\}_{m=1}^{M}$.

The selected relays $R_{(l)}, l \in \mathcal{J}$, first decode the data received from the $K$ selected sources using the maximum likelihood (ML) detector as follows

$$
\hat{\epsilon}_{S_{(k)} R_{(l)}}=\underset{\epsilon_{S_{(k)}}}{\arg \min _{q}}\left\{\left|y_{S_{(k)} R_{(l)}}-\sqrt{\rho} h_{S_{(k)} R_{(l)}} x_{S_{(k)}}\right|^{2}\right\},
$$

and then sequentially transmit their network-coded symbols to the destination. The NC operation is applied to all correct or incorrect received symbols [14]. In particular, relay $R_{(l)}$ linearly combines estimated symbols in $\mathbb{F}_{q}$ using the weighting coefficients $\alpha_{S_{(k)} R_{(l)}}$ forming an MDS code. MDS codes always exist if the field size is sufficiently large and are proven to be maximal-diversity-achievable in the uplink multiple-source, multiple-relay cooperative systems. Such network codes satisfy the Singleton bound and minimize the total number of packets required at the destination to decode the sources' packets. The network-coded symbol generated by relay $R_{(l)}$ can then be written as

$$
\hat{\epsilon}_{R_{(l)}}=\sum_{k \in \mathcal{I}} \bigoplus\left(\alpha_{S_{(k)} R_{(l)}} \bigotimes \hat{\epsilon}_{S_{(k)} R_{(l)}}\right) .
$$

Modulating $\hat{\epsilon}_{R_{(l)}}$ to $\hat{x}_{R_{(l)}}$, the received signal from relay $R_{(l)}, l \in \mathcal{J}$, at $D$ can be expressed as

$$
y_{R_{(l)} D}=\sqrt{\rho} h_{R_{(l)} D} \hat{x}_{R_{(l)}}+w_{R_{(l)} D}
$$

Fig. 2 shows an example of GURS NCC scheme when $N=$ $5, K=3, M=3, L=2, \gamma_{S_{5} D}>\gamma_{S_{2} D}>\gamma_{S_{1} D}>\gamma_{S_{3} D}>$ $\gamma_{S_{4} D}, \mathcal{I}=\{1,4,5\}, \gamma_{2 \mid\{3,5,4\}}^{\min }>\gamma_{3 \mid\{3,5,4\}}^{\min }>\gamma_{1 \mid\{3,5,4\}}^{\min }$, and $\mathcal{J}=\{1,3\}$.

\section{Outage Probability}

In this section, we derive closed-form expression for the OP of GURS NCC system, assuming n.i.i.d. Rayleigh fading channels over all wireless channels.

The following lemma is of important when it provides the closed-form expression of the OP.

Lemma 1. Let $X_{1}, X_{2}, \ldots, X_{n}$ be $n$ independent and nonidentical RVs with probability density function (PDF) $f_{X_{i}}\left(x_{i}\right)=\lambda_{i} e^{-\lambda_{i} x_{i}}$. Then,

$$
\operatorname{Pr}\left\{X_{1}>X_{2}>\ldots>X_{n}\right\}=\prod_{v=2}^{n}\left[\frac{\lambda_{v}}{\lambda_{1}+\sum_{i=2}^{v} \lambda_{i}}\right] .
$$

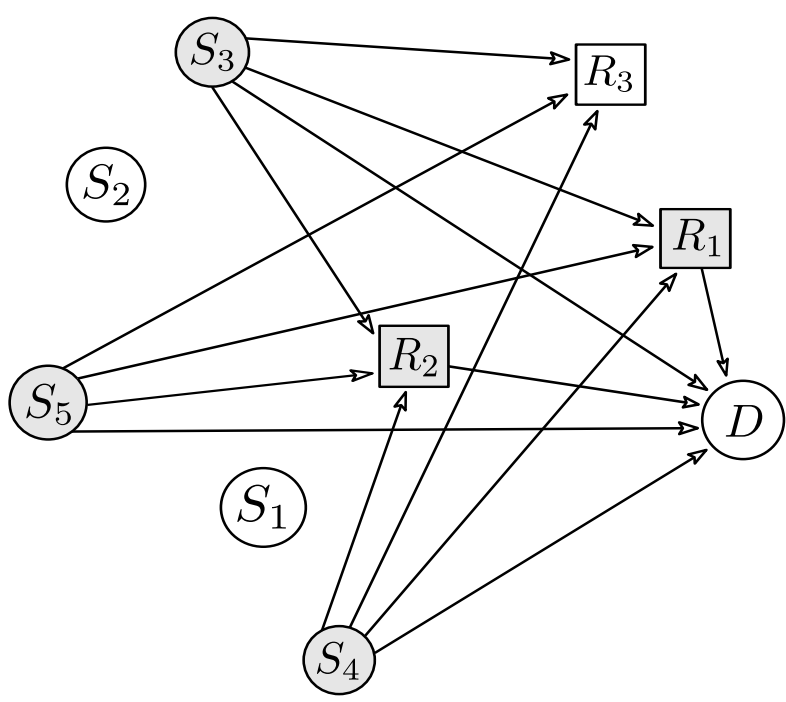

Fig. 2. System model for GURS NCC scheme. Example with $N=5, K=3$, $M=3, L=2, \gamma_{S_{5} D}>\gamma_{S_{2} D}>\gamma_{S_{1} D}>\gamma_{S_{3} D}>\gamma_{S_{4} D}, \mathcal{I}=\{1,4,5\}$, $\gamma_{2 \mid\{3,5,4\}}^{\min }>\gamma_{3 \mid\{3,5,4\}}^{\min }>\gamma_{1 \mid\{3,5,4\}}^{\min }$, and $\mathcal{J}=\{1,3\}$.

Proof. The proof is by induction.

- Base Case: The probability in (9) can be written in the integral form as

$$
\begin{aligned}
& \operatorname{Pr}\left\{X_{1}>X_{2}>\ldots>X_{n}\right\}= \\
& \underbrace{\int_{0}^{\infty} \int_{0}^{x_{1}} \int_{0}^{x_{2}} \ldots \int_{0}^{x_{n-1}}}_{n} \prod_{i=1}^{n}\left[f_{X_{i}}\left(x_{i}\right)\right] d x_{n} d x_{n-1} \ldots d x_{1} .
\end{aligned}
$$

We begin by verifying equation (9) for $n=2$. For $n=2$, (10) can be obtaied as

$$
\begin{aligned}
\operatorname{Pr}\left\{X_{1}>X_{2}\right\} & =\int_{0}^{\infty} \int_{0}^{x_{1}} \lambda_{1} e^{-\lambda_{1} x_{1}} \lambda_{2} e^{-\lambda_{2} x_{2}} d x_{2} d x_{1} \\
& =\int_{0}^{\infty} \lambda_{1} e^{-\lambda_{1} x_{1}}\left(1-e^{-\lambda_{2} x_{1}}\right) d x_{1} \\
& =\frac{\lambda_{2}}{\lambda_{1}+\lambda_{2}}
\end{aligned}
$$

which verifies that (9) is true for $n=2$.

- Induction Hypothesis: Assume that (9) holds when $n=$ $k$ i.e.,

$$
\operatorname{Pr}\left\{X_{1}>X_{2}>\ldots>X_{k}\right\}=\prod_{v=2}^{k}\left[\frac{\lambda_{v}}{\lambda_{1}+\sum_{i=2}^{v} \lambda_{i}}\right] .
$$

- Inductive Step: Now, we need to prove that (9) holds when $n=k+1$ using the assumption in (12):

$$
\begin{aligned}
& \operatorname{Pr}\left\{X_{1}>X_{2}>\ldots>X_{k+1}\right\}= \\
& \operatorname{Pr}\left\{X_{1}>X_{2}>\ldots>X_{k}\right\} \underbrace{\operatorname{Pr}\left\{X_{k+1}<X_{1}, X_{2}, \ldots, X_{k}\right\}}_{\mathcal{B}} .
\end{aligned}
$$


The probability $\mathcal{B}$ in (13) can be derived as follows

$$
\begin{aligned}
\mathcal{B} & =\underbrace{\int_{0}^{\infty} \int_{x_{k+1}}^{\infty} \int_{x_{k+1}}^{\infty} \ldots \int_{x_{k+1}}^{\infty} \prod_{i=1}^{k+1}\left[f_{X_{i}}\left(x_{i}\right)\right] d x_{1} \ldots d x_{k+1}}_{k+1} \\
& =\lambda_{k+1} \int_{0}^{\infty} e^{-\left(\lambda_{1}+\lambda_{2}+\ldots+\lambda_{k+1}\right) x_{k+1}} d x_{k+1} \\
& =\frac{\lambda_{k+1}}{\lambda_{1}+\lambda_{2}+\ldots .+\lambda_{k+1}} .
\end{aligned}
$$

Substituting (14) into (13), we have

$$
\begin{aligned}
& \operatorname{Pr}\left\{X_{1}>X_{2}>\ldots>X_{k+1}\right\} \\
& =\prod_{v=2}^{k}\left[\frac{\lambda_{v}}{\lambda_{1}+\sum_{i=2}^{v} \lambda_{i}}\right]\left[\frac{\lambda_{k+1}}{\lambda_{1}+\lambda_{2}+\ldots+\lambda_{k+1}}\right] \\
& =\prod_{v=2}^{k+1}\left[\frac{\lambda_{v}}{\lambda_{1}+\sum_{i=2}^{v} \lambda_{i}}\right]
\end{aligned}
$$

Thus, (9) holds for $n=k+1$, and the proof of the induction step is complete.

By the principle of induction, (9) is true for all $n \geq 2$ which concludes the proof $^{5}$.

Special Case 1. For the special case of i.i.d. RVs i.e., $\lambda_{i}=\lambda$, $\forall i$, (9) is simplified to

$$
\operatorname{Pr}\left\{X_{1}>X_{2}>\ldots>X_{n}\right\}=\frac{1}{n !} .
$$

Theorem 1. Consider a cooperative network that consists of $N$ users, $M$ relays, and one destination. Assume the relays use $\mathrm{DF}$ protocol and apply $\mathrm{NC}$ on the received users' symbols. If the destination selects the $i_{1}^{\text {th }}, i_{2}^{\text {th }}, \ldots, i_{K}^{\text {th }}$ best users and the $j_{1}^{\text {th }}, j_{2}^{\text {th }}, \ldots, j_{L}^{\text {th }}$ best relays, the OP of the system when $K>L$ can be formulated as

$$
\mathcal{P}_{\text {out }_{1}}=\sum_{\eta=0}^{K-L-1} \operatorname{Pr}\left\{\mathcal{E}_{\eta}\right\}+\sum_{\eta=1}^{L}\left(\operatorname{Pr}\left\{\mathcal{E}_{K-\eta}\right\} \sum_{\ell=0}^{\eta-1} \operatorname{Pr}\left\{\mathcal{V}_{\ell}\right\}\right)
$$

On the other hand, the OP when $K \leq L$ is given by

$$
\mathcal{P}_{\text {out }_{2}}=\sum_{\eta=1}^{K}\left(\operatorname{Pr}\left\{\mathcal{E}_{K-\eta}\right\} \sum_{\ell=0}^{\eta-1} \operatorname{Pr}\left\{\mathcal{V}_{\ell}\right\}\right)
$$

where $\operatorname{Pr}\left\{\mathcal{E}_{\eta}\right\}$ and $\operatorname{Pr}\left\{\mathcal{V}_{\ell}\right\}$ are, respectively, given by (19) and (20), as shown on the top of the next page, in which

$$
\operatorname{Pr}\left\{\mathcal{O}_{S_{n} D}\right\}=1-e^{-\lambda_{S_{n} D} \gamma_{t h}},
$$

with $\lambda_{i j}=\frac{1}{\rho \sigma_{i j}^{2}}$.

Furthermore, $\operatorname{Pr}\left\{\mathcal{O}_{m} \mid \mathcal{A}\right\}$ is given by

$$
\operatorname{Pr}\left\{\mathcal{O}_{m} \mid \mathcal{A}\right\}=1-e^{-\lambda_{m \mid \mathcal{A}} \gamma_{t h}},
$$

${ }^{5}$ We note that the result in Lemma 1 can be directly obtained using $n$ fold integrals given by (10). The direct proof of the obtained result, however, requires lengthy mathematical manipulations that do not bring much insight. In the interest of space, we chose to provide the shorter proof based on induction. In doing so, we first derived the results for small values of $n$ using (10). We noted that with mathematical manipulations they can be represented in the simple form of (9), where we proved (9) by induction. with $\lambda_{m \mid \mathcal{A}}$ being

$$
\lambda_{m \mid \mathcal{A}}=\lambda_{S_{\left(i_{1}\right)} R_{m}}+\ldots+\lambda_{S_{\left(i_{K}\right)} R_{m}}+\lambda_{R_{m} D} .
$$

Proof. In GURS NCC, the destination receives $K+L$ packets; $K$ packets from selected users and $L$ network-coded packets from selected relays. Due to the severe channel fading some of the links might be in outage and thereby only a subset of packets can be successfully recovered by the destination. If the destination receives at least $K$ error-free packets, either from the selected users or from the selected relays, it is capable of recovering $K$ original packets; otherwise, an outage occurs ${ }^{6}$.

The overall OP of GURS NCC thus depends upon the outage events of direct user-to-destination links and dualhop indirect user-to-relay-to-destination links. Let $\mathcal{E}_{\eta}$ and $\mathcal{V}_{\ell}$ denote the set of non-outage selected users and relays with cardinality $\eta$ and $\ell$, respectively. Mathematically, $\mathcal{E}_{\eta}$ and $\mathcal{V}_{\ell}$ can be written, respectively, as

$$
\begin{gathered}
\mathcal{E}_{\eta} \triangleq\left\{S_{(k)} \in \mathcal{S}: \gamma_{S_{(k)} D}>\gamma_{t h}\right\}, \\
\mathcal{V}_{\ell} \mid \mathcal{A} \triangleq\left\{R_{(l)} \in \mathcal{R}: \gamma_{(l) \mid \mathcal{A}}^{\min }>\gamma_{t h}\right\} .
\end{gathered}
$$

where $\gamma_{t h}$ is the threshold SNR.

The overall outage events of GURS NCC can then be expressed as

$$
\mathcal{O}=\mathcal{O}^{\prime} \bigcup \mathcal{O}^{\prime \prime}
$$

where $\mathcal{O}^{\prime}$ corresponds to the outage events when $K>L$ and there are not enough non-outage selected users, $\eta$, such that even if $\ell=L$, the destination is still in outage i.e., $\eta<K-L$. On the other hand, $\mathcal{O}^{\prime \prime}$ stands for the outage events where $\eta \geq K-L$, but the sum of non-outage selected users and relays is less than $K$ i.e., $\eta+\ell<K$.

Now, we proceed to obtain $\operatorname{Pr}\left\{\mathcal{E}_{\eta}\right\}$ and $\operatorname{Pr}\left\{\mathcal{V}_{\ell}\right\}$, based on which, $\operatorname{Pr}\left\{\mathcal{O}^{\prime}\right\}$ and $\operatorname{Pr}\left\{\mathcal{O}^{\prime \prime}\right\}$ can be derived.

An outage event occurs in a given link when its corresponding instantaneous SNR falls below $\gamma_{t h}$. The threshold SNR $\gamma_{t h}$ can be written in terms of the transmission rate $R_{0}$ (in bits per channel use) as $\gamma_{t h}=2^{R_{0}}-1$. Let $\mathcal{O}_{i j}$ denote the outage event of link $i \rightarrow j$. The OP of $i \rightarrow j$ link can be then written as

$$
\operatorname{Pr}\left\{\mathcal{O}_{i j}\right\}=\operatorname{Pr}\left\{\gamma_{i j}<\gamma_{t h}\right\},
$$

where $\gamma_{i j}=\rho\left|h_{i j}\right|^{2}$ is the instantaneous SNR of link $i \rightarrow j$. Noting that $\gamma_{i j}$ is exponentially distributed, (27) is readily solved as

$$
\operatorname{Pr}\left\{\mathcal{O}_{i j}\right\}=1-\int_{\gamma_{t h}}^{\infty} \lambda_{i j} e^{-\lambda_{i j} y} d y=1-e^{-\lambda_{i j} \gamma_{t h}},
$$

Applying order statistics properties and using (28), $\operatorname{Pr}\left\{\mathcal{E}_{\eta}\right\}$ can be written as (19).

On the other hand, $\operatorname{Pr}\left\{\mathcal{V}_{\ell}\right\}$ can be formulated using total probability theorem as

$$
\operatorname{Pr}\left\{\mathcal{V}_{\ell}\right\}=\sum_{\mathcal{A}} \operatorname{Pr}\left\{\mathcal{V}_{\ell} \mid \mathcal{A}\right\} \operatorname{Pr}\{\mathcal{A}\}
$$

${ }^{6} \mathrm{We}$ assume that the network codes are constructed over a sufficiently large field size $q$. This ensures that the resulting network-coded messages are distinct and mutually independent. The destination thus requires only $K$ messages (either from selected users or from selected relays) to jointly recover $K$ original messages. 


$$
\begin{aligned}
& \operatorname{Pr}\left\{\mathcal{E}_{\eta}\right\}=\sum_{v=N-i_{\eta+1}+1}^{N-i_{\eta}}\left(\sum_{\substack{a_{1}, \cdots, a_{v} \in\{1, \ldots, N\} \\
a_{1} \neq \cdots \neq a_{v}}}\left[\prod_{n=a_{1}}^{a_{v}} \operatorname{Pr}\left\{\mathcal{O}_{S_{n} D}\right\} \prod_{\substack{n^{\prime}=1 \\
n^{\prime} \neq\left\{a_{1}, \ldots, a_{v}\right\}}}^{N}\left(1-\operatorname{Pr}\left\{\mathcal{O}_{S_{n^{\prime}} D}\right\}\right)\right]\right) . \\
& \operatorname{Pr}\left\{\mathcal{V}_{\ell}\right\}=\sum_{\mathcal{A}}\left(\sum_{\substack{v=j_{\ell+1}+1 \\
a_{1}, \ldots, a_{i} \in\{1, \ldots, M\} \\
a_{1} \neq \ldots \neq a_{v}}}^{M-j_{\ell}}\left[\prod_{m=a_{1}}^{a_{v}} \operatorname{Pr}\left\{\mathcal{O}_{m} \mid \mathcal{A}\right\} \prod_{\substack{m^{\prime}=1 \\
m^{\prime} \neq\left\{a_{1}, \ldots, a_{v}\right\}}}^{M}\left(1-\operatorname{Pr}\left\{\mathcal{O}_{m^{\prime}} \mid \mathcal{A}\right\}\right)\right]\right) \\
& \left.\times \sum_{\substack{z_{1}, \ldots, z_{N} \in\{1, \ldots, N\} \\
z_{1} \neq \ldots \neq z_{N} \\
z_{i_{1}}, \ldots, z_{i_{K}} \in \mathcal{A}}}\left(\prod_{n=2}^{N}\left[\frac{\lambda_{S_{z_{n}} D}}{\lambda_{S_{z_{1}} D}+\sum_{i=2}^{n} \lambda_{S_{z_{i}} D}}\right]\right)\right)
\end{aligned}
$$

where the sum spans over all $\left(\begin{array}{l}N \\ K\end{array}\right)$ possible $\mathcal{A}$ 's from the set of $N$ candidate users and $\operatorname{Pr}\left\{\mathcal{V}_{\ell} \mid \mathcal{A}\right\}$ is given by (30), as shown on the top of this page. Further, $\operatorname{Pr}\{\mathcal{A}\}$ can be derived as (31) using Lemma 1. Note that the sum in (31) spans over $(N-K) ! K$ ! possibilities. For the special case of $\lambda_{S_{n} D} \approx \lambda_{S D}$, $\forall n$, we have $\operatorname{Pr}\{\mathcal{A}\} \approx \frac{1}{\left(\begin{array}{c}N \\ K\end{array}\right)}$.

$$
\operatorname{Pr}\{\mathcal{A}\}=\sum_{\substack{z_{1}, \ldots, z_{N} \in\{1, \ldots, N\} \\ z_{1} \neq \ldots \neq z_{N} \\ z_{i_{1}}, \ldots, z_{i_{K}} \in \mathcal{A}}}\left(\prod_{n=2}^{N}\left[\frac{\lambda_{S_{z_{n}} D}}{\lambda_{S_{z_{1}} D}+\sum_{i=2}^{n} \lambda_{S_{z_{i}} D}}\right]\right) .
$$

Now, plugging (30) and (31) into (29), the closed-form expression of $\operatorname{Pr}\left\{\mathcal{V}_{\ell}\right\}$ can be obtained as (20).

Finally, using (19), (20), and (26) one can obtain the closedform expression for the OP as given by (17) and (18). Thus, we complete the proof.

Special Case 2. The derived OP expression in (17) and (18) is based on the assumption of asymmetric n.i.i.d. Rayleigh fading channels over all wireless links and can be treated as the generalized versions of semi-symmetric n.i.i.d. channels or symmetric i.i.d. channels ${ }^{7}$. Furthermore, it subsumes all existing results in the literature as special cases. In particular, for $K=N$ and $L=M$ (NCC without user-relay selection) and asymmetric n.i.i.d. channels, it reduces to (34) in [15]. When $K=N$ and $L$ highest-SNR relays are selected, it reduces to (21) and (22) in [15]. For i.i.d. channels with

\footnotetext{
${ }^{7}$ Semi-symmetric n.i.i.d. subchannels refers to the case when $\lambda_{S_{n} D} \approx$ $\lambda_{S D}, \forall n$, and $\lambda_{R_{m} D} \approx \lambda_{R D}, \forall m$. This assumption can be applicable to cooperative uplink cellular systems [20], [28] where the mobile users and relays are formed as clusters. On the other hand, the channels are said symmetric i.i.d. when $\lambda_{S_{n} D}=\lambda_{R_{m} D}=\lambda_{S_{n} R_{m}}, \forall n, m$.
}

$K=N$ and any arbitrary relay selection, it coincides to (4) and (7) in [26].

\section{Asymptotic AnAlysis}

In this section, we first derive the asymptotic outage expression at the high SNR regime to quantify the achievable diversity order and coding gain. Then, we provide some insights and guidelines that can be drawn from our diversity analysis.

\section{A. Asymptotic Outage Probability}

In the previous section, closed-form expression of the OP has been derived, which is still too complicated to learn the relationship between OP and different system parameters. To gain deeper insights about how the system parameters impact on the outage performance, we now characterize the asymptotic behavior of the OP in the high-SNR regime. From the asymptotic expression, we extract two important system design parameters, namely the diversity order and the coding gain.

Theorem 2. Consider a cooperative network that consists of $N$ users, $M$ relays, and one destination. Assume the relays use DF protocol and apply NC on the received users' symbols. If the destination selects the $i_{1}^{\text {th }}, i_{2}^{\text {th }}, \ldots, i_{K}^{\text {th }}$ best users and the $j_{1}^{\text {th }}, j_{2}^{\text {th }}, \ldots, j_{L}^{\text {th }}$ best relays, the achievable diversity order when $K>L$ can be obtained as

$$
G_{d_{1}}=\left\{\begin{array}{cc}
N-i_{K-L}+1, & \\
\psi_{L}^{\max }<M+i_{K-L}+1 \\
N+M-\psi_{L}^{\max }+2, \\
\psi_{L}^{\max }>M+i_{K-L}+1
\end{array}\right.
$$


and the the coding gain is given by

$$
G_{c_{1}}=\left\{\begin{array}{l}
\frac{\Psi_{1}^{\prime-\frac{1}{N-i} K-L^{+1}}}{\gamma_{t h}}, \\
\frac{\Psi_{1}^{\prime \prime} \frac{1}{N+M-\psi_{L}^{\max }+2}}{\gamma_{t h}}, \\
\psi_{L}^{\max }<M+i_{K-L}+1 \\
\frac{\left(\Psi_{1}^{\prime}+\Psi_{1}^{\prime \prime}\right)^{-\frac{1}{N-i} K-L+1}}{\gamma_{t h}}, \\
\psi_{L}^{\max }=M+i_{K-L}+1
\end{array}\right.
$$

where $\psi_{\delta}^{\max }=\max \left\{\psi_{\eta}\right\}_{\eta=1}^{\delta}$ with $\psi_{\eta}=i_{K-\eta+1}+j_{\eta}$. Further,

$$
\Psi_{1}^{\prime}=\sum_{\substack{a_{1}, \cdots, a_{N-i} a+1 \\ a_{1} \neq \cdots \neq a_{N-i_{K-L}+1}}}\left(\prod_{n=a_{1}}^{a_{N-i_{K}-L}+1}\left(\frac{1}{\sigma_{S_{n} D}^{2}}\right)\right),
$$

and

$$
\Psi_{1}^{\prime \prime}=\sum_{\eta: \psi_{\eta}=\psi_{L}^{\max }} Q_{\eta}
$$

where $Q_{q}$ is given by (36) on the top of the next page and $\frac{1}{\sigma_{m \mid \mathcal{A}}^{2}}=\frac{1}{\sigma_{S_{\left(i_{1}\right)} R_{m}}^{2}}+\ldots+\frac{1}{\sigma_{S_{\left(i_{K}\right)} R_{m}}^{R^{2}}}+\frac{1}{\sigma_{R_{m} D}^{2}}$.

On the other hand, the achievable diversity order and the coding gain when $K \leq L$ can be obtained as

$$
G_{d_{2}}=N+M-\psi_{K}^{\max }+2
$$

and

$$
G_{c_{2}}=\frac{\Psi_{2}^{-\frac{1}{N+M-\psi_{K}^{\max }+2}}}{\gamma_{t h}}
$$

where

$$
\Psi_{2}=\sum_{\eta: \psi_{\eta}=\psi_{K}^{\max }} Q_{\eta}
$$

Proof. In asymptotically high-SNR regime, the OP can be written as

$$
\lim _{\rho \rightarrow \infty} \mathcal{P}_{\text {out }} \stackrel{\rho \rightarrow \infty}{\approx}\left(G_{c} \cdot \rho\right)^{-G_{d}}
$$

where the variable $G_{d}$ in (40) denotes the diversity order and determines the slope of the OP curve. This is given by

$$
G_{d}=-\lim _{\rho \rightarrow \infty} \frac{\log \left(\mathcal{P}_{\text {out }}\right)}{\log (\rho)} .
$$

On the other hand, $G_{c}$ represents the coding gain and quantify the SNR advantage of the asymptotic OP with respect to the reference curve $\rho^{-G_{d}}$.

To find the asymptotic expressions in the high SNR regime, we use Taylor series expansion of the exponential function given by $e^{-x}=\sum_{k=0}^{\infty} \frac{(-x)^{k}}{k !}$. Plugging this expression in (21), we have

$$
\operatorname{Pr}^{\infty}\left\{\mathcal{O}_{S_{n} D}\right\}=\lambda_{S_{n} D} \gamma_{t h}
$$

Similarly, (22) can be approximated as

$$
\operatorname{Pr}^{\infty}\left\{\mathcal{O}_{m} \mid \mathcal{A}\right\}=\lambda_{m \mid \mathcal{A}} \gamma_{t h}
$$

Substituting (42) and (43) into (19) and (20), and then keeping dominant terms, we respectively have

$$
\operatorname{Pr}^{\infty}\left\{\mathcal{E}_{\eta}\right\}=\sum_{\substack{a_{1}, \cdots, a_{N-i_{\eta+1}+1} \\ a_{1} \neq \cdots \neq a_{N-i_{\eta+1}+1}}}\left(\prod_{n=a_{1}}^{a_{N-i_{\eta+1}+1}} \lambda_{S_{n} D} \gamma_{t h}\right),
$$

and

$$
\begin{aligned}
\operatorname{Pr}^{\infty}\left\{\mathcal{V}_{\ell}\right\} & =\sum_{\mathcal{A}}\left(\sum_{\substack{a_{1}, \cdots, a_{M-j_{\ell+1}+1} \\
a_{1} \neq \ldots \neq a_{M-j_{\ell+1}+1}}} \prod_{m=a_{1}}^{a_{M-j_{\ell+1}+1}} \lambda_{m \mid \mathcal{A}} \gamma_{t h}\right. \\
& \left.\times \sum_{\substack{z_{1}, \ldots, z_{N} \\
z_{1} \neq \ldots \neq z_{N} \\
z_{i_{1}}, \ldots, z_{i_{K}} \in \mathcal{A}}}^{N}\left(\prod_{n=2}^{N}\left[\frac{\lambda_{S_{z_{n}} D}}{\lambda_{S_{z_{1}} D}+\sum_{i=2}^{n} \lambda_{S_{z_{i}} D}}\right]\right)\right)
\end{aligned}
$$

By plugging (44), (45) into (17) and then retaining the dominant terms, (17) can be approximated as

$\mathcal{P}_{\text {out }_{1}}^{\infty}=\operatorname{Pr}^{\infty}\left\{\mathcal{E}_{K-L-1}\right\}+\sum_{\eta: \eta=\eta_{L}^{\max }} \operatorname{Pr}^{\infty}\left\{\mathcal{E}_{K-\eta}\right\} \operatorname{Pr}^{\infty}\left\{\mathcal{V}_{\eta-1}\right\}$

Now, based on the relationship between $\psi_{L}^{\max }$ and $M+i_{K-L}+$ 1, (46) in high SNRs can be derived as follows:

- Case 1: $\psi_{L}^{\max }<M+i_{K-L}+1$. In this case, $\mathcal{P}_{\text {out }_{1}}^{\infty}$ is determined by the first term in (46) and is given by

$$
\mathcal{P}_{\text {out }_{1}}^{\infty}=\Psi_{1}^{\prime}\left(\frac{\gamma_{t h}}{\rho}\right)^{N-i_{K-L}+1} .
$$

- Case 2: $\psi_{L}^{\max }>M+i_{K-L}+1$. In this case, $\mathcal{P}_{\text {out }_{1}}^{\infty}$ is determined by the second term in (46) and can be expressed as

$$
\mathcal{P}_{\text {out }_{1}}^{\infty}=\Psi_{1}^{\prime \prime}\left(\frac{\gamma_{t h}}{\rho}\right)^{N+M-\psi_{L}^{\max }+2} .
$$

- Case 3: $\psi_{L}^{\max }=M+i_{K-L}+1$. In this case, $\mathcal{P}_{\text {out }_{1}}^{\infty}$ is determined by the first and second terms in (46). The asymptotic outage expression can then be written as

$$
\mathcal{P}_{\text {out }_{1}}^{\infty}=\left(\Psi_{1}^{\prime}+\Psi_{1}^{\prime \prime}\right)\left(\frac{\gamma_{t h}}{\rho}\right)^{N-i_{K-L}+1}
$$

Finally, using (40), the diversity order and coding gain of GURS NCC system when $K>L$ are, respectively, given by (32) and (33).

Now, we proceed to obtain the asymptotic outage expression for $K \leq L$. Substituting (44), (45) into (18) and then retaining the dominant terms, (18) in high SNRs can be written as

$$
\mathcal{P}_{\text {out }_{2}}^{\infty}=\Psi_{2}\left(\frac{\gamma_{t h}}{\rho}\right)^{N+M-\psi_{K}^{\max }+2} .
$$

Based on (40) and (50), the achievable diversity order and the coding gain when $K \leq L$ are, respectively, given by (37) and (38). This concludes the proof.

Special Case 3. The derived diversity order in (32) and (37) is the most generic expression in the literature and includes all 


$$
\begin{aligned}
& Q_{\eta}=\sum_{\substack{a_{1}, \cdots, a_{N-i_{K-\eta+1}+1} \\
a_{1} \neq \cdots \neq a_{N-i_{K-\eta+1}+1}}} \prod_{n=a_{1}}^{a_{N-i_{K}}-\eta+1+1}\left(\frac{1}{\sigma_{S_{n} D}^{2}}\right) \sum_{\mathcal{A}}\left(\sum_{\substack{a_{1}, \cdots, a_{M-j_{\eta}+1} \\
a_{1} \neq \ldots \neq a_{M-j_{\eta}+1}}} \prod_{m=a_{1}}^{a_{M-j_{\eta}+1}}\left(\frac{1}{\sigma_{m \mid \mathcal{A}}^{2}}\right)\right. \\
& \left.\times \sum_{\substack{z_{1}, \ldots, z_{N} \in\{1, \ldots, N\} \\
z_{1} \neq \ldots \neq z_{N} \\
z_{i_{1}}, \ldots, z_{i} \in \mathcal{A}}} \prod_{n=2}^{N}\left[\frac{\lambda_{S_{z_{n}} D}}{\lambda_{S_{z_{1}} D}+\sum_{i=2}^{n} \lambda_{S_{z_{i}} D}}\right]\right) .
\end{aligned}
$$

existing results as special cases. More specifically, for $K=N$ and $L=M$, it reduces to $G_{d}=M+1$ [7], [8], [14], [15]. When $K=N$ and $L$ highest-SNR relays are selected, the diversity order for $K>L$ and $K \leq L$ reduces to $G_{d_{1}}=L+1$ and $G_{d_{2}}=M+1$. This coincides with the diversity order reported in [14], [15]. When $K=N$ and any arbitrary relays are selected, it reduces to the diversity order of $G_{d_{1}}=L+1$ and $G_{d_{2}}=N+M-j_{N}+1$ for $K>L$ and $K \leq L$, respectively [26]. Finally, when $K$ best users and $L$ best relays are selected, it coincides to the results in [20].

Special Case 4. The derived diversity order can also be thought as a generalization of the all results available in the non-NCC literature. For $K=1$ and $M=0$ (non-cooprative multiuser case), the diversity order reduces to $G_{d_{1}}=N-i_{1}+1$ which coincides to that of [23]. For $N=1$ and the best relay selection, it reduces to $G_{d_{2}}=M+1$ [29]. For $N=1$ and the $j_{1}^{\text {th }}$ best relay is selected, it reduces to $G_{d_{2}}=M-j_{1}+2$ which agrees with that of in [24]. When the best user and the best relay are selected, it reduces to $G_{d_{2}}=N+M$. This diversity order is identical to that of [17], [18]. For $N=1$ and any arbitrary relay selection, it reduces to $G_{d_{2}}=M-j_{1}+2$. This result is in agreement with the diversity order reported in [27].

\section{B. Insights and Guidelines}

Here, we provide some insights and guidelines that can be drawn from our diversity analysis and can help the understanding and the design of practical NCC systems with user-relay selection protocols.

From (32), the following remarks and guidelines can be drawn:

Remark 1. From $\left(\begin{array}{c}N \\ K\end{array}\right)\left(\begin{array}{c}M \\ L\end{array}\right)$ different user-relay selections, $\Delta_{1}=$ $N-K+1$ distinct diversity orders can be achieved. Therefore, the number of achievable diversity orders is a function of the number of users $N$, number of selected users $K$, but it is independent of the number of relays $M$ and the number of selected relays $L$.

Remark 2. The maximum and minimum diversity orders are given by

$$
\left\{\begin{array}{l}
G_{d_{1}}^{\max }=N-K+L+1, \\
G_{d_{1}}^{\min }=L+1
\end{array}\right.
$$

The condition $i_{K-L}=K-L$ is the necessary (but not sufficient) condition for achieving maximum diversity $G_{d_{1}}^{\max }$.
Other user selections with $i_{K-L} \neq K-L$ cannot provide $G_{d_{1}}^{\max }$. The condition $i_{K-L}=K-L$ is satisfied if and only if the set of selected users includes $K-L$ highest-SNR users. On the other hand, the system has the minimum diversity $G_{d_{1}}^{\text {min }}$ if $i_{K-L}=N-L$ or $\psi_{L}^{\max }=N+M-L+1$.

Remark 3. When $\psi_{L}^{\max }<M+i_{K-L}+1$, the diversity is determined by $G_{d_{1}}=N-i_{K-L}+1$ which only depends on the number of users $N$ and the $i_{K-L}^{\text {th }}$ best user. This indicates that other system parameters such as number of relays $M$, number of selected relays $L$, and any arbitrary relay selection do not impact the achievable diversity order.

Remark 4. The number of user selections that always guarantee the diversity order of $G_{d_{1}}=N-i_{K-L}+1$, no matter how the RS proceeds, can be expressed as

$$
\xi=\left(\begin{array}{c}
i_{K-L}-1 \\
K-L-1
\end{array}\right)
$$

If user selection includes $K-L$ highest-SNR users, we have $i_{K-L}=K-L$ which yields the maximum diversity order of $G_{d_{1}}^{\max }=N-K+L+1$ and $\xi=1$. This implies that only one user selection always guarantees maximum diversity $G_{d_{1}}^{\max }$, no matter which of $L$ relays are selected. This user selection is indeed the best user selection that includes $K$ highest-SNR users. On the other hand, when $i_{K-L}=N-$ $L$, we have the minimum diversity order of $G_{d_{1}}^{\min }=L+1$ and $\xi=\left(\begin{array}{l}N-L-1 \\ K-L-1\end{array}\right)$. This suggests that $\left(\begin{array}{l}N-L-1 \\ K-L-1\end{array}\right)$ number of user selections, including the worst user selection, have always minimum diversity $G_{d_{1}}^{\min }$ irrespective of RS process. Note that the order of the selected relays only manifests its effect on the coding gain, rather than the diversity. According to (33), if the set of selected relays does not include the lowest-SNR relay (i.e., $j_{L} \neq M$ ), the coding gain of any arbitrary relay selection is identical to that of the best relay selection leading to the same outage performance in the high-SNR regime.

Remark 5. When $\psi_{L}^{\max }>M+i_{K-L}+1$, the diversity is determined by $G_{d_{1}}=N+M-\psi_{L}^{\max }+2$. In this case, the diversity is a function of the number of users $N$, number of relays $M$, the $j_{1}^{\text {th }}, j_{2}^{\text {th }}, \ldots, j_{L}^{\text {th }}$ best relays and the $i_{K-L+1}^{\text {th }}, i_{K-L+2}^{\text {th }}, \ldots, i_{K}^{\text {th }}$ best users. This implies that all the system parameters impact the achievable diversity except the $i_{1}^{\text {th }}, i_{2}^{\text {th }}, \ldots, i_{K-L}^{\text {th }}$ best users.

Example 1. Consider a network with $N=6, K=4, M=4$, and $L=2$. These system parameters satisfy the condition $K>L$. All $\left(\begin{array}{l}6 \\ 4\end{array}\right)\left(\begin{array}{l}4 \\ 2\end{array}\right)=90$ user-relay selections with their 
TABLE I

Diversity Orders for All Possible User-Relay Selections: $N=6, K=4, M=4, L=2$.

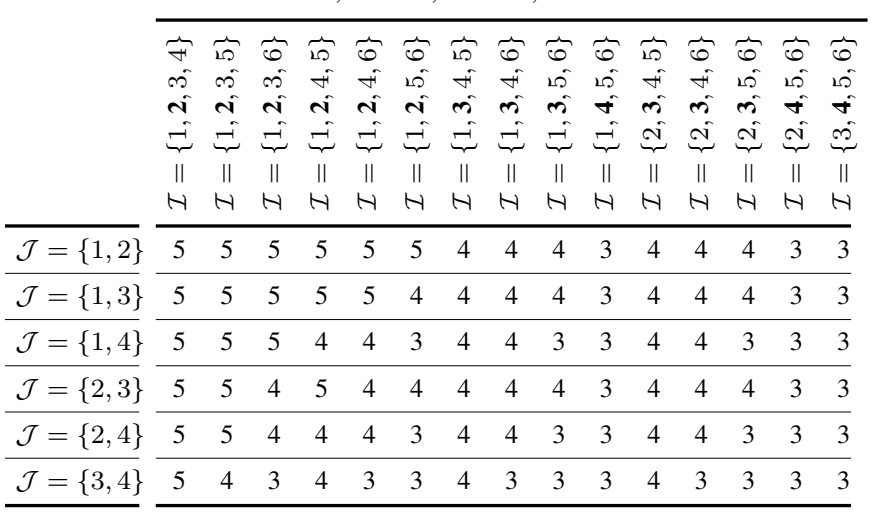

corresponding achievable diversity orders (32) are reported in Table I. As can be seen, there are $\Delta_{1}=N-K+1=3$ different diversity orders ranging from minimum diversity $G_{d_{1}}^{\min }=L+1=3$ to maximum diversity $G_{d_{1}}^{\max }=N-$ $K+L+1=5$. This confirms the statements in Remarks 1,2 . In addition, the user selections with $i_{2} \neq 2$ i.e., $i_{2}=3,4$ are not capable of achieving the maximum diversity of five even if the best relay selection is performed, confirming Remark 2 . Furthermore, there are six user selections that always achieve the diversity order of $G_{d_{1}}=N-i_{K-L}+1$, no matter which of two relays are selected. More precisely, the best user selection $\mathcal{I}=\{1,2,3,4\}$ guarantees the maximum diversity of five. There are also $\xi=\left(\begin{array}{l}2 \\ 1\end{array}\right)=2$ (52) number of user selections that always achieve the diversity of four i.e., $\mathcal{I}=\{1,3,4,5\}$ and $\mathcal{I}=\{2,3,4,5\}$. Besides, $\xi=\left(\begin{array}{l}3 \\ 1\end{array}\right)=3$ user selections always have the minimum diversity of three. They are $\mathcal{I}=\{1,4,5,6\}$, $\mathcal{I}=\{2,4,5,6\}$ and the worst user selection $\mathcal{I}=\{3,4,5,6\}$. This confirms the statements in Remark 4.

On the other hand, based on (37), we have the following design insights and remarks.

Remark 6. All possible user-relay selections provide $\Delta_{2}=$ $N+M-K-L+1$ different diversities. Accordingly, the number of diversity orders is a function of all system parameters.

Remark 7. The diversity order depends on $N, M$, the $i_{1}^{\text {th }}, i_{2}^{\text {th }}, \ldots, i_{K}^{\text {th }}$ best users and the $j_{1}^{\text {th }}, j_{2}^{\text {th }}, \ldots, j_{K}^{\text {th }}$ best relays. Therefore, the $j_{K+1}^{\text {th }}, j_{2}^{\text {th }}, \ldots, j_{L}^{\text {th }}$ best relays do not change the achievable diversity order.

Remark 8. The maximum and minimum diversity orders are given by (53). The diversity order of $G_{d_{2}}^{\max }=N-K+M+1$ can be achieved if and only if $\psi_{K}^{\max }=K+1$, implying that the set of selected users and relays must include $K$ highestSNR users and $K$ highest-SNR relays. On the other hand, the system has the minimum diversity order of $G_{d_{2}}^{\min }=L+1$ if and only if $\psi_{K}^{\max }=N+M-L+1$.

$$
\left\{\begin{array}{l}
G_{d_{2}}^{\max }=N-K+M+1, \\
G_{d_{2}}^{\min }=L+1
\end{array}\right.
$$

TABLE II

Diversity Orders for All Possible User-Relay SElections: $N=4, K=2, M=6, L=4$.

\begin{tabular}{|c|c|c|c|c|c|c|c|c|c|c|c|c|c|c|c|}
\hline 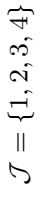 & 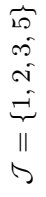 & 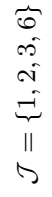 & 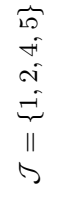 & 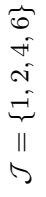 & 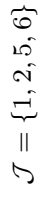 & 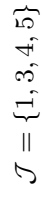 & 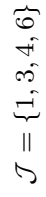 & 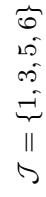 & 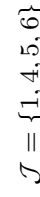 & $\underbrace{2}_{0}$ & & 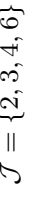 & 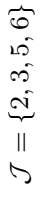 & 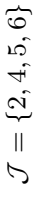 & 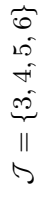 \\
\hline 9 & 9 & 9 & 9 & 9 & 9 & 8 & 8 & 8 & 7 & 8 & & 8 & 8 & 7 & 7 \\
\hline 8 & 8 & 8 & 8 & 8 & 8 & 8 & 8 & 8 & 7 & 7 & & 7 & 7 & 7 & 6 \\
\hline 7 & 7 & 7 & 7 & 7 & 7 & 7 & 7 & 7 & 7 & 6 & & 6 & 6 & 6 & 5 \\
\hline 8 & 8 & 8 & 8 & 8 & 8 & 7 & 7 & 7 & 6 & 7 & & 7 & 7 & 6 & 6 \\
\hline 7 & 7 & 7 & 7 & 7 & 7 & 7 & 7 & 7 & 6 & 6 & & 6 & 6 & 6 & 5 \\
\hline 7 & 7 & 7 & 7 & 7 & 7 & 6 & 6 & 6 & 5 & 6 & & 6 & 6 & 5 & 5 \\
\hline
\end{tabular}

Remark 9. The number of relay selections that achieve full diversity order $G_{d_{2}}^{\max }$ is given by

$$
\zeta=\left(\begin{array}{c}
M-K \\
L-K
\end{array}\right)
$$

Remark 10. The relay selections that include $K$ highestSNR relays has the minimum diversity of $M+1$ when the lowest-SNR user is in the set of selected users or equivalently $i_{K}=N$. Similarly, the worst relay selection has the minimum diversity of $L+1$ when $i_{K}=N$.

Remark 11. The term $N-K+1$ in $G_{d_{v}}^{\max }(v=1,2)$ (51) and (53) corresponds to the MUD and the remaining terms $L$ and $M$ correspond to the $\mathrm{CD}$. It can be readily checked that $G_{d_{v}}^{\max }$ increases when the number of users $N$ increases. Further, it decreases when the number of selected users $K$ increases. Obviously, when $K=N$ the MUD gain vanishes and only the CD gain can be achieved [20].

Example 2. Now, consider a network with $N=4, K=2$, $M=6$, and $L=4$. These system parameters satisfy the condition $K \leq L$. The achievable diversity orders (37) for all $\left(\begin{array}{l}4 \\ 2\end{array}\right)\left(\begin{array}{l}6 \\ 4\end{array}\right)=90$ user-relay selections are provided in Table II. It can be seen that there are $\Delta_{2}=N+M-K-L+1=5$ different achievable diversity orders; from the minimum diversity of $G_{d_{2}}^{\min }=L+1=5$ to the maximum diversity of $G_{d_{2}}^{\max }=N-K+M+1=9$ i.e., $G_{d_{2}}=5,6,7$, 8, 9. This confirms Remarks 6, 8. Besides, $\zeta=\left(\begin{array}{l}4 \\ 2\end{array}\right)=6$ (54) relay selections achieve maximum diversity $G_{d_{2}}^{\max }=9$. Furthermore, the relay selections that include $K=2$ highestSNR relays have the minimum diversity of $M+1=7$ when $i_{2}=4$ i.e., $\mathcal{I}=\{1,4\}, \mathcal{I}=\{2,4\}$, and $\mathcal{I}=\{3,4\}$. Similarly, the worst relay selection $\mathcal{J}=\{3,4,5,6\}$ has the minimum diversity of $L+1=5$ when $i_{2}=4$. This confirms the statements in Remarks 9 and 10.

\section{NumericAl Results ANd Discussions}

In this section, we provide numerical and simulation results to verify the derived analytical expressions. Table III shows the simulation parameters. 
TABLE III

Simulation Parameters

\begin{tabular}{c|lc}
\hline Notation & Parameter & Value \\
\hline$R_{0}$ & Transmission rate & $\{1,2\}$ \\
\hline$N$ & Number of users & $\{4,6\}$ \\
\hline$M$ & Number of relays & $\{4,6\}$ \\
\hline$K$ & Number of selected users & $\{2,4\}$ \\
\hline$L$ & Number of selected relays & $\{2,4\}$ \\
\hline
\end{tabular}

\section{A. Number of Selected Users Greater Than Number of Se- lected Relays}

Here, we consider the same system parameters as in Table I. Specifically, we assume that number of users $N=6$, number of selected users $K=4$, number of relays $M=4$, and number of selected relays $L=2$. The transmission rate $R_{0}$ is set one. The randomly generated values for the channel variances of the user-to-relay links are given in (55). Note that the effect of different transmit power, noise variance, and path loss on the received signal can be lumped into the fading variances [2]. In particular, the element at the $n^{\text {th }}$ row and the $m^{\text {th }}$ column of matrix $\Sigma_{S R}$ corresponds to the variance of the channel between user $S_{n}$ and relay $R_{m}$.

$$
\boldsymbol{\Sigma}_{S R}=\left[\begin{array}{llll}
2.3 & 2.6 & 0.5 & 5.1 \\
6.5 & 0.8 & 4.9 & 6.5 \\
4.8 & 2.7 & 5.1 & 1.3 \\
3.9 & 3.9 & 0.7 & 5.2 \\
3.7 & 4.2 & 2.1 & 1.1 \\
2.6 & 1.9 & 3.5 & 4.3
\end{array}\right] .
$$

Furthermore, the $n^{\text {th }}$ and $m^{\text {th }}$ elements in the vector variances $\boldsymbol{\Sigma}_{S D}$ (56) and $\boldsymbol{\Sigma}_{R D}$ (57) correspond to the channel variance of the link from user $S_{n}$ and Relay $R_{m}$ to the destination $D$.

$$
\begin{gathered}
\boldsymbol{\Sigma}_{S D}=\left[\begin{array}{llllll}
0.7 & 0.8 & 1.2 & 1.3 & 0.9 & 2.3
\end{array}\right], \\
\boldsymbol{\Sigma}_{R D}=\left[\begin{array}{llll}
2.3 & 1.2 & 3.8 & 4.9
\end{array}\right] .
\end{gathered}
$$

In what follows, the derived analytical expressions for the $\mathrm{OP}$ and diversity order are examined via representative numerical plots and Monte-Carlo simulations.

Fig. 3 plots the OP of GURS NCC versus SNR $\rho$ with different sets of selected users and two highest-SNR relays $(\mathcal{J}=\{1,2\})$. One can observe that the analytical curves perfectly match with simulation results, confirming the correctness of the derived expression (17). Furthermore, the asymptotic curves accurately predict the behaviour of the outage provability in the high-SNR regime. More specifically, the slope of the asymptotic curves reveals that the maximum diversity order of $G_{d_{1}}^{\max }=N-K+L+1=5$ can be achieved if $i_{2}=2$. This is verified as the diversity for $\mathcal{I}=\{1,2,3,5\}$ and $\mathcal{I}=\{1,2,4,6\}$ with $i_{2}=2$ is equal to $G_{d_{1}}^{\max }=5$, while that of $\mathcal{I}=\{1,3,4,5\}$ and $\mathcal{I}=\{1,4,5,6\}$ with $i_{2}=3$ and $i_{2}=4$ is equal to four and three, respectively. In conclusion, the condition $i_{K-L}=K-L$ is the necessary condition for achieving maximum diversity $G_{d_{1}}^{\max }$, as shown in Table I.

In Fig. 4, we plot the OP of GURS NCC for different userrelay selections. It can be seen, the best user selection with the highest- and lowest-SNR relays achieves the maximum diversity order of $G_{d_{1}}^{\max }=5$. When $\mathcal{I}=\{1,2,3,5\}$, however,

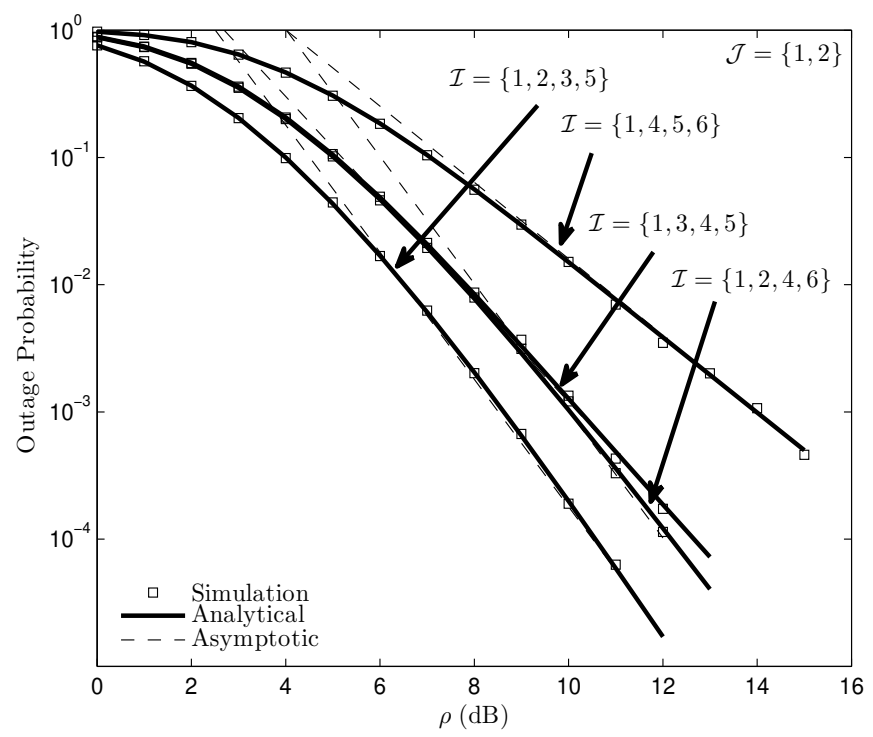

Fig. 3. OP versus $\rho$ for $N=6, K=4, M=4, L=2, \mathcal{J}=\{1,2\}$ with different user selections.

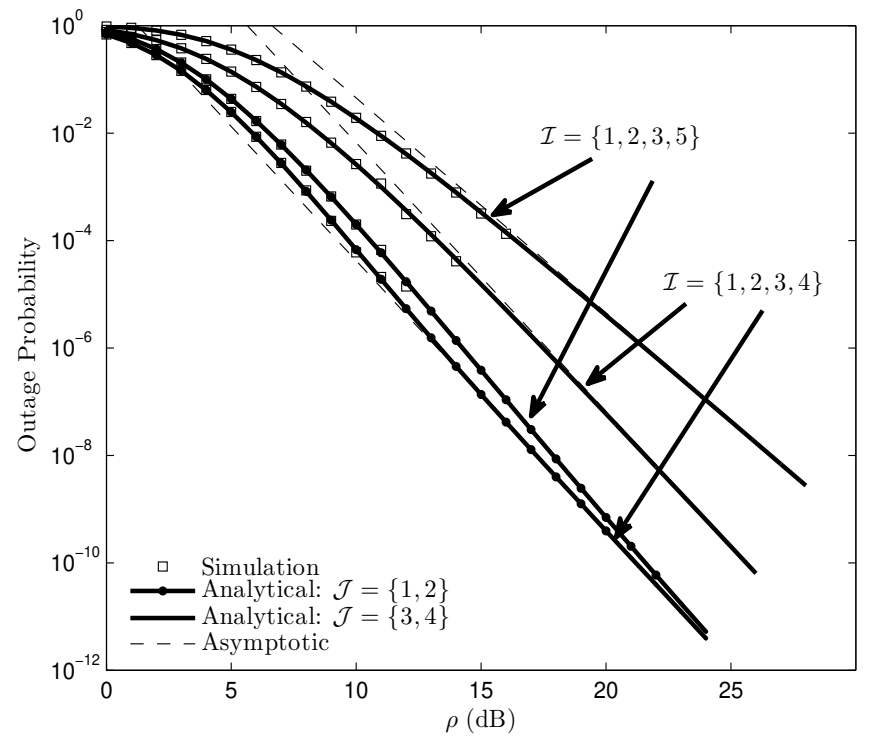

Fig. 4. OP versus $\rho$ for $N=6, K=4, M=4, L=2$ with different user-relay selections.

the diversity order varies depending on the relay selection process. More precisely, the diversity order for $\mathcal{I}=\{1,2,3,5\}$ with $\mathcal{J}=\{1,2\}$ is equal to five, while that of $\mathcal{I}=\{1,2,3,5\}$ with $\mathcal{J}=\{3,4\}$ is four. This indicates that the best user selection always guarantees the maximum diversity order $G_{d_{1}}^{\max }$, no matter how the relay selection proceeds, as shown in Table I.

Fig. 5 illustrates the outage performance of GURS NCC for $\mathcal{I}=\{2,4,5,6\}$ and different relay selections. As can be seen, the slope of the curves is always proportional to the number of users $N$ and the $i_{K-L}^{\mathrm{th}}$ best user which is reflected by the parallel slopes of the asymptotic lines. This verifies the theoretical observation that the diversity order is equal to $G_{d_{1}}=N-i_{K-L}+1=3$ (cf. Table I). Interestingly, this diversity is preserved, no matter which of two relays are 


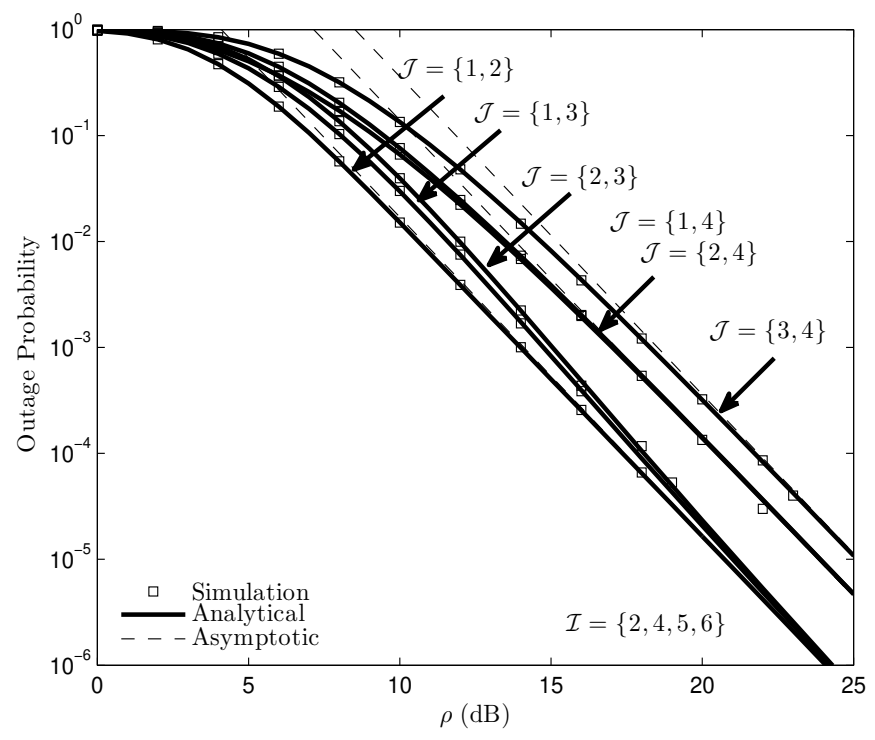

Fig. 5. OP versus $\rho$ for $N=6, K=4, M=4, L=2, \mathcal{I}=\{2,4,5,6\}$ and different relay selection sets.

selected. Furthermore, when the worst relay is not selected i.e., $j_{2} \neq 4$, the outage performance is exactly the same as that of the best relay selection in the high SNR regime. On the other hand, when the set of selected relays includes the worst relay $\left(j_{2}=4\right)$, the outage is always worse than that of the best relay selection both in finite and asymptotic SNRs.

\section{B. Number of Selected Users Less Than Number of Selected Relays}

Here, the same system parameters as in Table II are considered. In particular, we assume $N=4, K=2, M=6$, $L=4$. These system parameters satisfies the condition $K \leq L$. The variances of the user-to-relay, user-to-destination, and relay-to-destination channels are generated randomly and are, respectively, given by (58), (59), and (60). We set the transmission rate as $R_{0}=2$.

$$
\begin{gathered}
\boldsymbol{\Sigma}_{S R}=\left[\begin{array}{llllll}
0.5 & 1.3 & 1.4 & 2.1 & 0.7 & 1.9 \\
2.1 & 2.8 & 2.9 & 2.5 & 2.6 & 1.5 \\
2.8 & 0.9 & 1.8 & 0.6 & 1.3 & 1.4 \\
1.6 & 1.5 & 1.2 & 3.2 & 2.8 & 2.4
\end{array}\right], \\
\boldsymbol{\Sigma}_{S D}=\left[\begin{array}{llllll}
1.9 & 0.9 & 1.5 & 1.1
\end{array}\right], \\
\boldsymbol{\Sigma}_{R D}=\left[\begin{array}{llllll}
1.2 & 2.7 & 3.3 & 2.2 & 0.9 & 2.6
\end{array}\right] .
\end{gathered}
$$

Fig. 6 shows the outage performance of GURS NCC when the best/worst user and best/worst relay sets are selected. Once again, the analytical curves are confirmed by simulations to be exact and accurate. Furthermore, the asymptotic lines well approximate the exact curves in the high SNR regime. In addition, we can readily see that the best user-relay selection achieves the maximum diversity of $G_{d_{2}}^{\max }=N-K+M+1=$ 9. On the other hand, the worst user-relay selection has the minimum diversity of $G_{d_{2}}^{\min }=L+1=5$. Besides, although the best-user worst-relay selection outperforms the best-relay worst-user selection in terms of the coding gain,

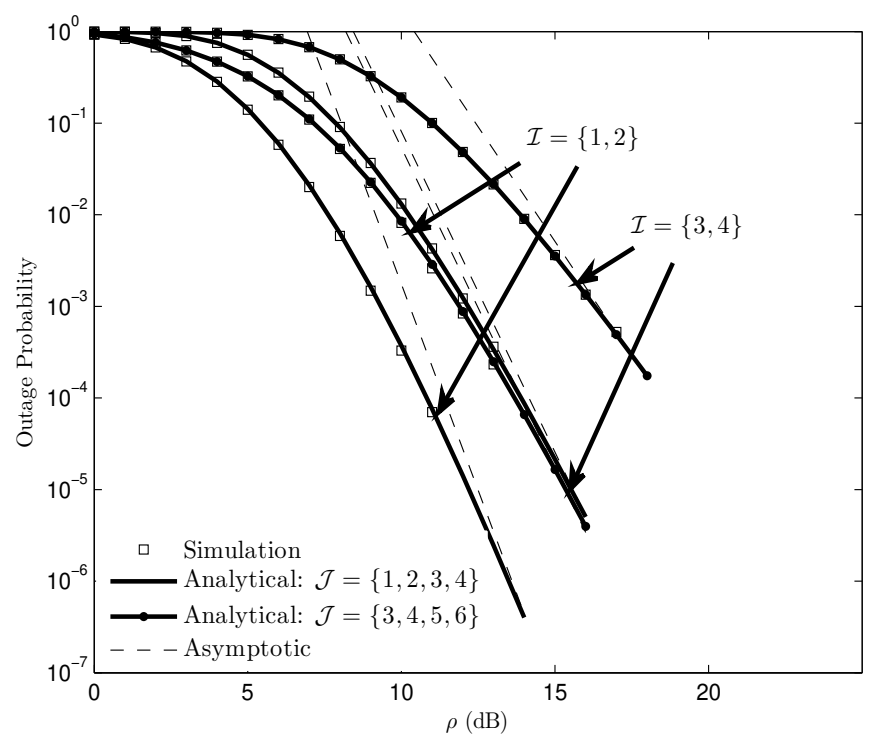

Fig. 6. OP versus $\rho$ for $N=4, K=2, M=6, L=4$ with the best/worst user selection and the best/worst relay selection.

the diversity order for both cases is identical and is equal to $G_{d_{2}}=M+1=7$ (cf. Table II).

Finally, Figs. 7 and 8 present the outage performance of GURS NCC for $\mathcal{J}=\{1,2,5,6\}$ and $\mathcal{J}=\{3,4,5,6\}$ when different sets of selected users are considered. As can be seen, three distinct diversities are achieved for $\mathcal{J}=\{1,2,5,6\}$ and $\mathcal{J}=\{3,4,5,6\}$ depending on the set of selected users. In particular, for $\mathcal{J}=\{1,2,5,6\}$, the maximum diversity of $G_{d_{2}}^{\max }=9$ is achieved when the user selection includes two highest-SNR users. Furthermore, when the user selection includes the worst user (i.e., $i_{2}=4$ ), the minimum diversity order of $G_{d_{2}}=M+1=7$ is achieved. Also, $\mathcal{I}=\{1,3\}$ and $\mathcal{I}=\{2,3\}$ have the diversity order of eight. On the other hand, from Fig. 8, we observe that in the case of $\mathcal{J}=\{3,4,5,6\}$ the diversity of $G_{d_{2}}=M+1=7$ is achieved for $\mathcal{I}=\{1,2\}$. Besides, when $i_{2}=4$ the diversity order of $G_{d_{2}}=L+1=5$ is achieved. For both $\mathcal{I}=\{1,3\}$ and $\mathcal{I}=\{2,3\}$ the diversity is identical and is equal to six. These observations are in agreement with the diversity orders reported in Table II.

\section{Conclusions}

In this paper, we proposed generalized user-relay selection in a multiuser multirelay NCC system. More specifically, we considered $N$ sources, $M$ relays, and a single destination. The destination selects the $i_{1}^{\text {th }}, i_{2}^{\text {th }}, \ldots, i_{K}^{\text {th }}$ best sources and the $j_{1}^{\text {th }}, j_{2}^{\text {th }}, \ldots, j_{L}^{\text {th }}$ best relays subject to any practical considerations. Considering n.i.i.d. Rayleigh fading channels, generalized closed-form expression for the OP has been derived. Asymptotic analysis has been further performed to quantify the diversity order and coding gain of the considered system. We showed that the derived diversity order is the generalized version of the earlier results reported in the literature and thus subsume all existing results as special cases. Our results also revealed that if $K>L, G_{d_{1}}=N-i_{K-1}+1$, and the set of selected relays does not include the lowest-SNR relay i.e., $j_{L} \neq M$, the coding gain of any arbitrary relay 


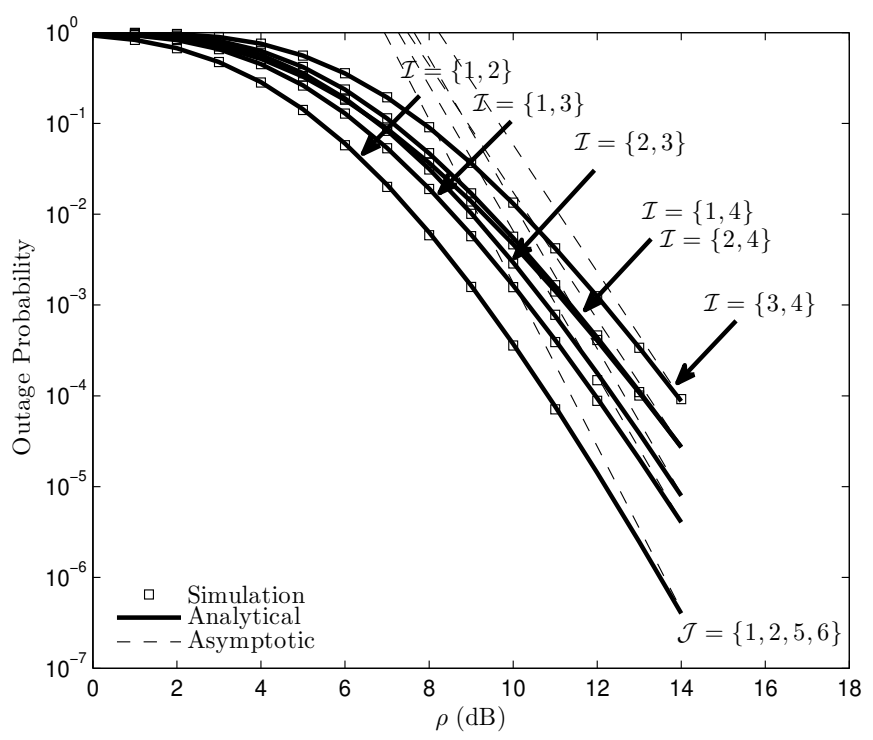

Fig. 7. OP versus $\rho$ for $N=4, K=2, M=6, L=4, \mathcal{J}=\{1,2,5,6\}$ and different user selections.

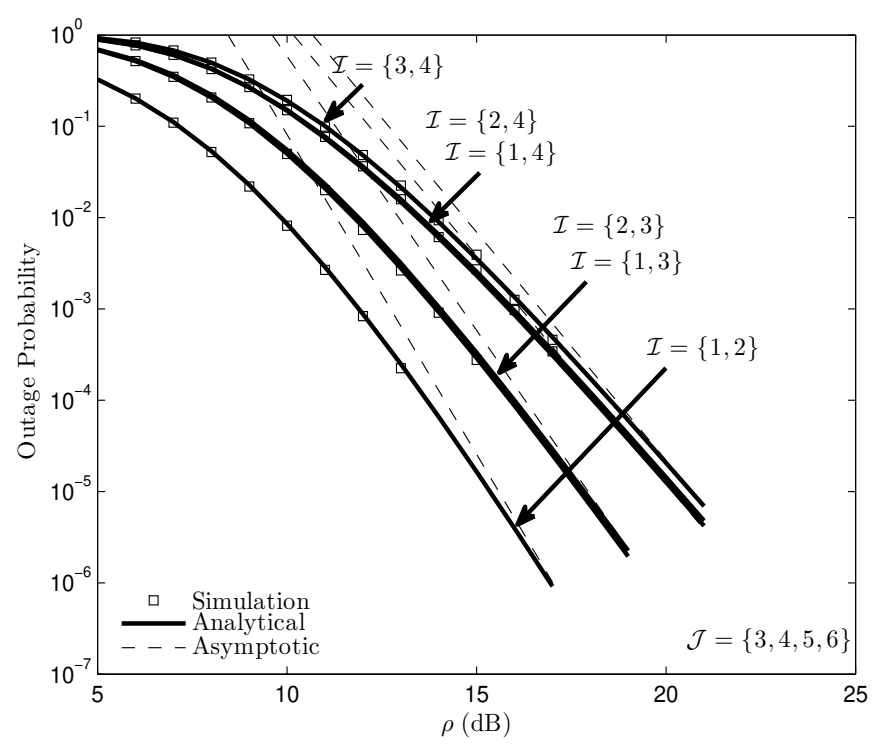

Fig. 8. OP versus $\rho$ for $N=4, K=2, M=6 L=4, \mathcal{J}=\{3,4,5,6\}$ and different user selections.

selection is identical to that of the best relay selection. On the other hand, when $K \leq L$ and the relay selections include $K$ highest-SNR relays, GURS NCC has the minimum diversity of $G_{d_{2}}=M+1$ when the lowest-SNR user is in the sets of selected users i.e., $i_{K}=N$. Similarly, the worst relay selection has the minimum diversity of $G_{d_{2}}^{\min }=L+1$ if $i_{K}=N$. Monte-Carlo simulations were also provided to corroborate the accuracy of our analytical findings. Our results clearly provide useful design insights and guidelines for practical cooperative systems with user-relay selection protocols.

The derived OP and diversity order expressions can be applied to investigate various new technologies, including energy harvesting and cognitive radio. Furthermore, the proposed GURS assumes perfect CSI for user-relay selection process. Hence, performance loss due to outdated CSI on the performance of GURS should be investigated. These tasks are set aside for future work.

\section{REFERENCES}

[1] M. Series, "IMT vision-framework and overall objectives of the future development of IMT for 2020 and beyond," Tech. Rep. Recommendation ITU-R M.2083-0, 2015.

[2] J. N. Laneman, D. N. C. Tse, and G. W. Wornell, "Cooperative diversity in wireless networks: Efficient protocols and outage behavior," IEEE Trans. Inf. Theory, vol. 50, no. 12, pp. 3062-3080, Dec. 2004.

[3] Q. Li, R. Q. Hu, Y. Qian, and G. Wu, "Cooperative communications for wireless networks: techniques and applications in LTE-advanced systems," IEEE Wireless Commun., vol. 19, no. 2, pp. 22-29, Apr. 2012.

[4] F. Boccardi, R. W. Heath, A. Lozano, T. L. Marzetta, and P. Popovski, "Five disruptive technology directions for 5G," IEEE Commun. Mag., vol. 52, no. 2, pp. 74-80, Feb. 2014.

[5] S. T. Başaran, G. Karabulut Kurt, M. Uysal, and I. Altunbaş, "A tutorial on network coded cooperation," IEEE Commun. Surveys Tuts., vol. 18, no. 4, pp. 2970-2990, 4th Quart. 2016.

[6] R. Ahlswede, N. Cai, S. Y. R. Li, and R. W. Yeung, "Network information flow," IEEE Trans. Inf. Theory, vol. 46, no. 4, pp. 1204 1216, Jul. 2000

[7] M. Xiao, J. Kliewer, and M. Skoglund, "Design of network codes for multiple-user multiple-relay wireless networks," IEEE Trans. Commun., vol. 60, no. 12, pp. 3755-3766, Dec. 2012.

[8] H. Topakkaya and Z. Wang, "Wireless network code design and performance analysis using diversity-multiplexing tradeoff," IEEE Trans. Commun., vol. 59, no. 2, pp. 488-496, Feb. 2011.

[9] A. R. Heidarpour, G. K. Kurt, and M. Uysal, "Finite-SNR diversitymultiplexing tradeoff for network coded cooperative OFDMA systems," IEEE Trans. Wireless Commun., vol. 16, no. 3, pp. 1385-1396, Mar. 2017.

[10] Z. C. Pereira, T. H. Ton, J. L. Rebelatto, R. D. Souza, and B. F. UchôaFilho, "Generalized network-coded cooperation in OFDMA communications," IEEE Access, vol. 6, pp. 6550-6559, Jan. 2018.

[11] A. R. Heidarpour, M. Ardakani, and C. Tellambura, "Network-coded cooperation with outdated CSI," IEEE Wireless Commun. Lett., vol. 22, no. 8, pp. 1720-1723, Aug. 2018.

[12] M. D. Renzo, M. Iezzi, and F. Graziosi, "On diversity order and coding gain of multisource multirelay cooperative wireless networks with binary network coding," IEEE Trans. Veh. Technol., vol. 62, no. 3, pp. 11381157, Mar. 2013.

[13] _ - "Error performance and diversity analysis of multi-source multirelay wireless networks with binary network coding and cooperative MRC," IEEE Trans. Wireless Commun., vol. 12, no. 6, pp. 2883-2903, Jun. 2013.

[14] M. D. Renzo, "On the achievable diversity of repetition-based and relay selection network-coded cooperation," IEEE Trans. Commun., vol. 62, no. 7, pp. 2296-2313, Jul. 2014.

[15] T. X. Vu, P. Duhamel, and M. D. Renzo, "On the diversity of networkcoded cooperation with decode-and-forward relay selection," IEEE Trans. Wireless Commun., vol. 14, no. 8, pp. 4369-4378, Aug. 2015.

[16] A. R. Heidarpour, M. Ardakani, C. Tellambura, and M. Di Renzo, "Relay selection in network-coded cooperative MIMO systems," IEEE Trans. Commun., vol. 67, no. 8, pp. 5346-5361, Aug. 2019.

[17] H. Ding, J. Ge, D. B. da Costa, and Z. Jiang, "A new efficient lowcomplexity scheme for multi-source multi-relay cooperative networks," IEEE Trans. Veh. Technol., vol. 60, no. 2, pp. 716-722, Feb. 2011.

[18] L. Sun, T. Zhang, L. Lu, and H. Niu, "On the combination of cooperative diversity and multiuser diversity in multi-source multi-relay wireless networks," IEEE Signal Process. Lett., vol. 17, no. 6, pp. 535-538, Jun. 2010.

[19] S. Chen, W. Wang, and X. Zhang, "Performance analysis of multiuser diversity in cooperative multi-relay networks under Rayleigh-fading channels," IEEE Trans. Wireless Commun., vol. 8, no. 7, pp. 3415-3419, Jul. 2009.

[20] A. R. Heidarpour, M. Ardakani, and C. Tellambura, "Multiuser diversity in network-coded cooperation: Outage and diversity analysis," IEEE Commun. Lett., vol. 23, no. 3, pp. 550-553, Mar. 2019.

[21] L. Fan, X. Lei, P. Fan, and R. Q. Hu, "Outage probability analysis and power allocation for two-way relay networks with user selection and outdated channel state information," IEEE Comm. Lett., vol. 16, no. 5, pp. 638-641, May 2012. 
[22] P. K. Sharma and P. K. Upadhyay, "Performance analysis of cooperative spectrum sharing with multiuser two-way relaying over fading channels," IEEE Trans. Veh. Technol., vol. 66, no. 2, pp. 1324-1333, Feb. 2017.

[23] A. M. Salhab and S. A. Zummo, "Multiuser cognitive networks with $N$ th-best user selection and imperfect channel estimation," Arab. J. Sci. Eng., vol. 39, no. 12, p. 8979-8987, Dec. 2014.

[24] S. S. Ikki and M. H. Ahmed, "On the performance of cooperativediversity networks with the $N$ th best-relay selection scheme," IEEE Trans. Commun, vol. 58, no. 11, pp. 3062-3069, Nov. 2010.

[25] L. Yang, K. Qaraqe, E. Serpedin, and X. Gao, "Performance analysis of two-way relaying networks with the $N$ th worst relay selection over various fading channels," IEEE Trans. Veh. Technol., vol. 64, no. 7, pp. 3321-3327, Jul. 2015.

[26] A. R. Heidarpour, M. Ardakani, and C. Tellambura, "Generalized relay selection for network-coded cooperation systems," IEEE Comm. Lett., vol. 21, no. 12, pp. 2742-2745, Dec. 2017.

[27] S.-I. Chu, "SER and PER performance of packet decode-and-forward relaying with generalized order selection combining," IEEE Wireless Commun. Lett., vol. 3, no. 5, pp. 449-452, Oct. 2014

[28] A. R. Heidarpour, M. Ardakani, C. Tellambura, and M. Di Renzo, "Generalized user-relay selection in network-coded cooperation systems," in Proc. IEEE Int. Conf. on Commun. (ICC), May 2019, pp. 1-6.

[29] A. Bletsas, A. Khisti, D. P. Reed, and A. Lippman, "A simple cooperative diversity method based on network path selection," IEEE J. Sel. Areas Commun., vol. 24, no. 3, pp. 659-672, 2006.

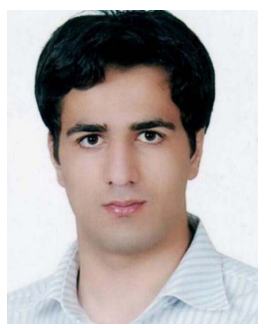

Ali Reza Heidarpour received the B.Sc. degree from University of Isfahan, Isfahan, Iran, in 2013 and the M.Sc. degree from Ozyegin University, Istanbul, Turkey, in 2016. He is currently working toward the $\mathrm{Ph}$.D. degree in electrical engineering at the University of Alberta, Edmonton, AB, Canada.

His research interests include cooperative communications, orthogonal frequency division multiplexing, and multiple-input multiple-output (MIMO) systems.

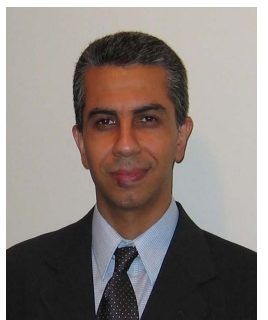

Masoud Ardakani (M'04-SM'09) received the B.Sc. degree from Isfahan University of Technology in 1994, the M.Sc. degree from Tehran University in 1997, and the Ph.D. degree from the University of Toronto, Canada, in 2004, all in Electrical Engineering. He was a Postdoctoral fellow at the University of Toronto from 2004 to 2005 . He is currently a Professor of Electrical and Computer Engineering at the University of Alberta, Canada. His research interests are in the general area of information theory. Dr. Ardakani serves as an Associate Editor at Large for the IEEE Transactions on Communications and has served as an Associate Editor for the IEEE Transactions on Communications and the IEEE Transactions on Wireless Communications, and as a senior editor for the IEEE Communications Letters.

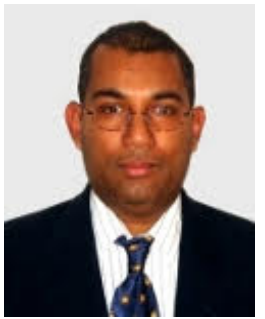

Chintha Tellambura (F'11) received the B.Sc. degree (with first-class honor) from the University of Moratuwa, Sri Lanka, the MSc degree in Electronics from King's College, University of London, United Kingdom, and the $\mathrm{PhD}$ degree in Electrical Engineering from the University of Victoria, Canada. He was with Monash University, Australia, from 1997 to 2002. Presently, he is a Professor with the Department of Electrical and Computer Engineering. University of Alberta. His current research interests include the design, modelling and analysis of current and future wireless networks. Prof. Tellambura served as an editor for both IEEE Transactions on Communications (1999-2011) and IEEE Transactions on Wireless Communications (2001-2007) and for the latter he was the Area Editor for Wireless Communications Systems and Theory during 2007-2012. He has received best paper awards in the Communication Theory Symposium in 2012 IEEE International Conference on Communications (ICC) in Canada and 2017 ICC in France. He is the winner of the prestigious McCalla Professorship and the Killam Annual Professorship from the University of Alberta. In 2011, he was elected as an IEEE Fellow for his contributions to physical layer wireless communication theory. In 2017, he was elected as a Fellow of Canadian Academy of Engineering. He has authored or coauthored over 500 journal and conference papers with an $\mathrm{H}$-index of 76 (Google Scholar).

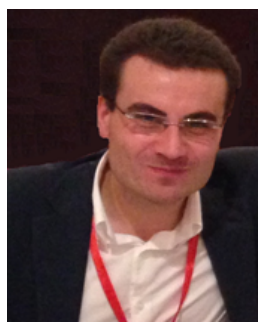

Marco Di Renzo (S'05-AM'07-M'09-SM'14-F'20) was born in L'Aquila, Italy, in 1978. He received the Laurea (cum laude) and Ph.D. degrees in electrical engineering from University of L'Aquila, Italy, in 2003 and 2007, respectively, and the Habilitation a Diriger des Recherches (Doctor of Science) degree from University Paris-Sud, France, in 2013.

Since 2010, he has been with the French National Center for Scientific Research (CNRS), where he is a CNRS Research Director (CNRS Professor) in the Laboratory of Signals and Systems (L2S) of ParisSaclay University - CNRS and CentraleSupelec, Paris, France. He is a Nokia Foundation Visiting Professor at Aalto University, Helsinki, Finland, and an Honorary Professor at University Technology Sydney, Sydney, Australia.

$\mathrm{He}$ serves as the Editor-in-Chief of IEEE Communications Letters. He served as an Editor of IEEE Transactions on Communications, IEEE Transactions on Wireless Communications, IEEE Communications Letters, and as the Associate Editor-in-Chief of IEEE Communications Letters. He is a Distinguished Lecturer of the IEEE Vehicular Technology Society and IEEE Communications Society.

$\mathrm{He}$ is a recipient of several awards, including the 2013 IEEE-COMSOC Best Young Researcher Award for Europe, Middle East and Africa, the 2013 NoE-NEWCOM\# Best Paper Award, the 2014-2015 Royal Academy of Engineering Distinguished Visiting Fellowship, the 2015 IEEE Jack Neubauer Memorial Best System Paper Award, the 2015 CNRS Award for Excellence in Research and Ph.D. Supervision, the 2016 MSCA Global Fellowship (declined), the 2017 SEE-IEEE Alain Glavieux Award, the 2018 IEEE-COMSOC Young Professional in Academia Award, the 2019 Nokia Foundation Distinguished Professorship, and 8 Best Paper Awards at IEEE conferences (2012 and 2014 IEEE CAMAD, 2013 IEEE VTC-Fall, 2014 IEEE ATC, 2015 IEEE ComManTel, 2017 IEEE SigTelCom, EAI 2018 INISCOM, IEEE ICC 2019).

He is a Highly Cited Researcher (Clarivate Analytics, Web of Science) and a Fellow of the IEEE. 


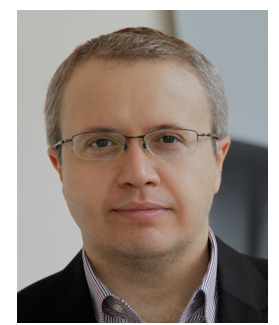

Murat Uysal received the B.Sc. and the M.Sc. degree in electronics and communication engineering from Istanbul Technical University, Istanbul, Turkey, in 1995 and 1998, respectively, and the $\mathrm{Ph} . \mathrm{D}$. degree in electrical engineering from Texas A\&M University, College Station, Texas, in 2001. $\mathrm{He}$ is currently a Full Professor and Chair of the Department of Electrical and Electronics Engineering at Ozyegin University, Istanbul, Turkey. He also serves as the Founding Director of the Center of Excellence in Optical Wireless Communicaion Technologies (OKATEM). Prior to joining Ozyegin University, he was a tenured Associate Professor at the University of Waterloo, Canada. Dr. Uysal's research interests are in the broad area of communication theory with a particular emphasis on the physical layer aspects of wireless communication systems in radio and optical frequency bands. On these topics, he has authored some 350 journal and conference papers and received more than 13.000 citations with an h-index of 56. Dr. Uysal is an IEEE Fellow and the former Chair of IEEE Turkey Section. He currently serves on the editorial board of IEEE Transactions on Wireless Communications. In the past, he served as an Editor for IEEE Transactions on Communications, IEEE Transactions on Vehicular Technology, and IEEE Communications Letters. He was involved in the organization of several IEEE conferences at various levels. In particular, he served as the Technical Program Committee Chair of major IEEE conferences including WCNC 2014, PIMRC 2019 and VTC-Fall 2019. Prof Uysal's major distinctions include NSERC Discovery Accelerator Award in 2008, University of Waterloo Engineering Research Excellence Award in 2010, Turkish Academy of Sciences Distinguished Young Scientist Award in 2011, Ozyegin University Best Researcher Award in 2014, National Instruments Engineering Impact Award in 2017, Elginkan Foundation Technology Award in 2018 and IEEE Communications Society Best Survey Paper Award in 2019 among others. 